Itinéraires Itinéraires

Littérature, textes, cultures

2016-2 | 2017

Livre, sérialité et transmédialité

\title{
In the dispositif / out of CTRL : netwars ou le hacking transmédia
}

In the Dispositif / Out of CTRL: netwars or the Transmedia Hacking

Karleen Groupierre et Anaïs Guilet

\section{OpenEdition}

Journals

Édition électronique

URL : http://journals.openedition.org/itineraires/3495

DOI : $10.4000 /$ itineraires.3495

ISSN : 2427-920X

Éditeur

Pléiade

Référence électronique

Karleen Groupierre et Anaïs Guilet, «In the dispositif / out of CTRL : netwars ou le hacking transmédia », Itinéraires [En ligne], 2016-2 | 2017, mis en ligne le 10 mai 2017, consulté le 03 octobre 2020. URL http://journals.openedition.org/itineraires/3495; DOI : https://doi.org/10.4000/itineraires.3495

Ce document a été généré automatiquement le 3 octobre 2020.

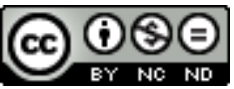

Itinéraires est mis à disposition selon les termes de la licence Creative Commons Attribution - Pas d'Utilisation Commerciale - Pas de Modification 4.0 International. 


\title{
In the dispositif / out of CTRL : netwars ou le hacking transmédia
}

\author{
In the Dispositif / Out of CTRL: netwars or the Transmedia Hacking \\ Karleen Groupierre et Anaïs Guilet
}

1 Le projet netwars, qui est, en 2017 encore en cours de production, est consacré à la cyber-criminalité. Il est composé de cinq éléments porteurs : un documentaire TV, un web-documentaire, une application de récit interactif (roman graphique), une succession de romans (audio, papier et e-book) et une série télévisée qui n'est pas encore sortie. netwars est produit par filmtank $\mathrm{GmbH}$ et réalisé par Michael Grotenhoff et Saskia Kress. Chaque média est également conçu sous la direction d'un auteurdirecteur, mais l'ensemble, ainsi qu'il est présenté sur le site officiel de l'univers du projet (http://netwars-project.com/), a été pensé et réalisé pour appartenir à un tout. Ces différents médias ont été conçus et produits dans le but d'alimenter un même univers narratif et le projet netwars peut donc être qualifié de transmédia tel que défini par Henry Jenkins, c'est-à-dire qu'il propose « une histoire qui se déploie au travers de multiples supports, chaque nouvel élément apportant une contribution caractéristique et précieuse à l'ensemble » (2006: 95-96).

2 Ainsi, les différents médias de netwars composent un univers commun entre réalité et fiction dans lequel le spectateur ${ }^{1}$ construit son propre récit et sa propre expérience en naviguant d'un média à un autre. Entre fonction vériste de la fiction et docufiction, netwars crée le trouble entre faits et fiction dans l'esprit de son spectateur. Cette ambiguïté, telle qu'elle est thématisée, esthétisée et structurellement créée par le dispositif transmédia est au centre de l'intérêt qu'il présente. Il faut alors noter que netwars se développe depuis 2015, soit au cœur de l'affaire Snowden (2013-...), grâce à laquelle le monde découvre l'ampleur du cyber-espionnage et sa dimension étatique. Dans un tel contexte, l'aspect paranogène du projet prend toute sa signification et l'enjeu sera d'expliquer comment celle-ci se construit de manière d'autant plus forte du fait de la qualité transmédia de netwars. La paranoïa, cet effet de psychose, " caractérisée par la présence d'idées délirantes systématisées et permanentes, surtout à thème de persécution» (Larousse) est en effet au centre du projet. Elle est le fruit 
d'un trouble entre imaginaire et réel. Dans netwars, la paranoïa ne provient pas tant du sujet en lui-même que du dispositif fictionnel qui mêle faits et éléments non référentiels ainsi que de la structure transmédia, dans sa dimension immersive et totalisante. Avec netwars tout est processus de hacking, c'est-à-dire recherche des failles et des vulnérabilités dans le but d'en tirer profit : le documentaire est hacké par la fiction, comme le spectateur par le dispositif transmédia.

Dans netwars, faits et fiction sont intriqués au point de créer un espace paranogène nourri des théories du complot. La paranoïa contamine l'expérience spectatorielle du fait de plusieurs facteurs: la partie fictionnelle du projet devient intrusive pour l'usager, le spectateur est noyé dans un flot de discours faussement contradictoires, il a l'illusion de faire des choix et l'impression de se forger son propre avis. Face à l'univers paranogène de netwars, comme l'internaute à l'égard de ses données sur le Web, l'objectif reste donc de parvenir à garder le contrôle. Ainsi, nous sommes tous constamment en prise avec Big Brother, marronnier avec lequel le personnage principal du web-documentaire ne se prive d'ailleurs pas d'ironiser : «Big Brother is watching you et tout ça ${ }^{2}$ !» Cependant, le tour de force du projet est de ne pas se contenter d'aborder la question de la surveillance sur le Web à partir des incontournables, voire des clichés du genre : 1984, la cinquième colonne et autres mythes conspirationnistes, mais de créer du fait de sa qualité transmédia un véritable dispositif panoptique au sens large qu'en donne Foucault. Car l'œuvre, à l'instar de beaucoup de transmédias ${ }^{3}$, se construit en forme de silo (Ertzscheid 2012), favorisant de ce fait une distribution fermée sur elle-même, centrifuge. Une forme qui pourrait être généralisée à bon nombre d'autres projets transmédias, mais que la dimension paranogène de netwars révèle de manière aiguë.

\section{Le complot netwars : entre faits et fiction?}

L'univers netwars propose une expérience à mi-chemin entre réalité et fiction. Les différents médias qui le constituent sont autonomes en même temps qu'ils permettent un enrichissement mutuel et global. Ainsi, chaque élément, qu'il relève du registre factuel ou fictionnel, contribue à la visée générale de cette œuvre plurielle.

\section{Documentaire, documenteur, docufiction?}

5 L'univers netwars mêle des faits et des éléments fictionnels. Leur distinction peut paraitre claire dans un premier temps du fait de la généricité des différents éléments transmédias: le web-documentaire et le documentaire télévisé ainsi que l'onglet «facts » du site web ou la veille effectuée sur le fil du compte Twitter du projet, sont du côté de la visée documentaire et un mandat plus fictionnel est attribué à l'application du roman graphique, au livre audio, à l'e-book et au livre papier. 
Fig. 1. Représentation de l'équilibre entre faits et fictions annoncé pour les différents médias de netwars

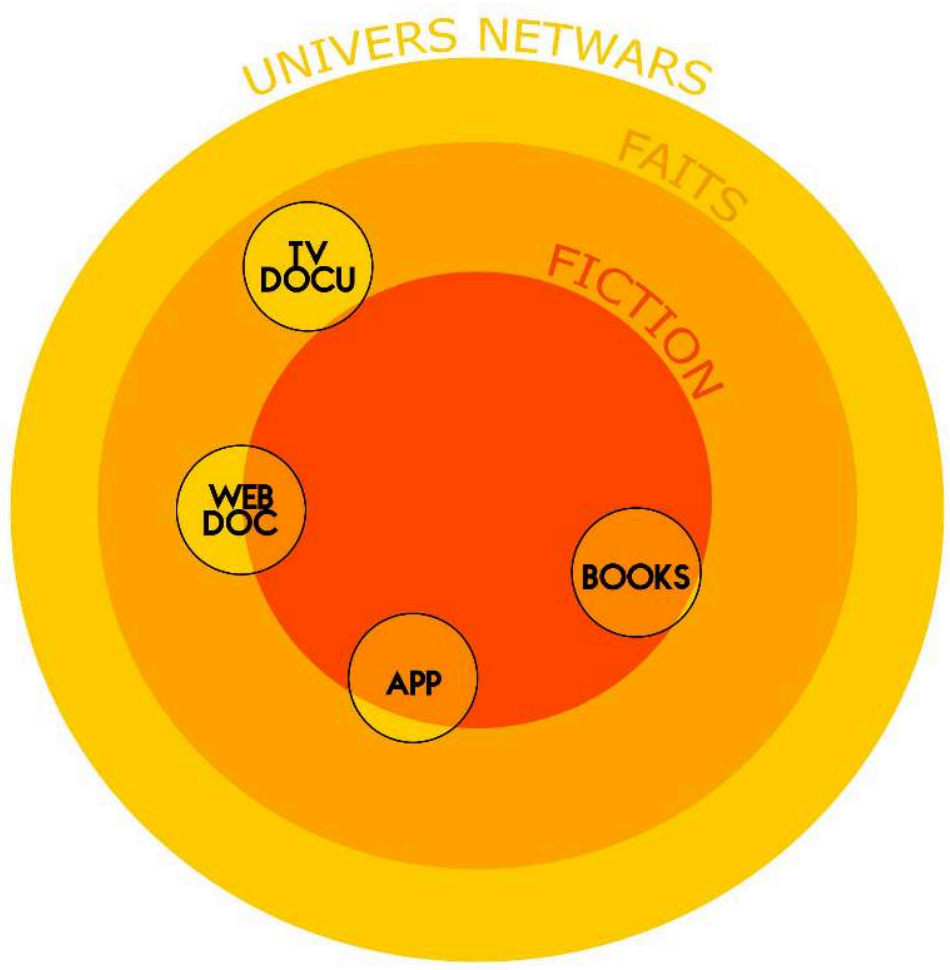

6 Toutefois, au sein de chacun des médias, tout n'est pas toujours aussi séparé. Ainsi, le roman graphique propose ponctuellement des hyperliens qui permettent d'accéder à des informations, des chiffres et autres données à valeur documentaire. 
Fig. 2. Sean Coleman, Felix Mertikat, Verena Klinke et al., 2015, The Butterfly Attack, filmtank GmbH. Application roman graphique.

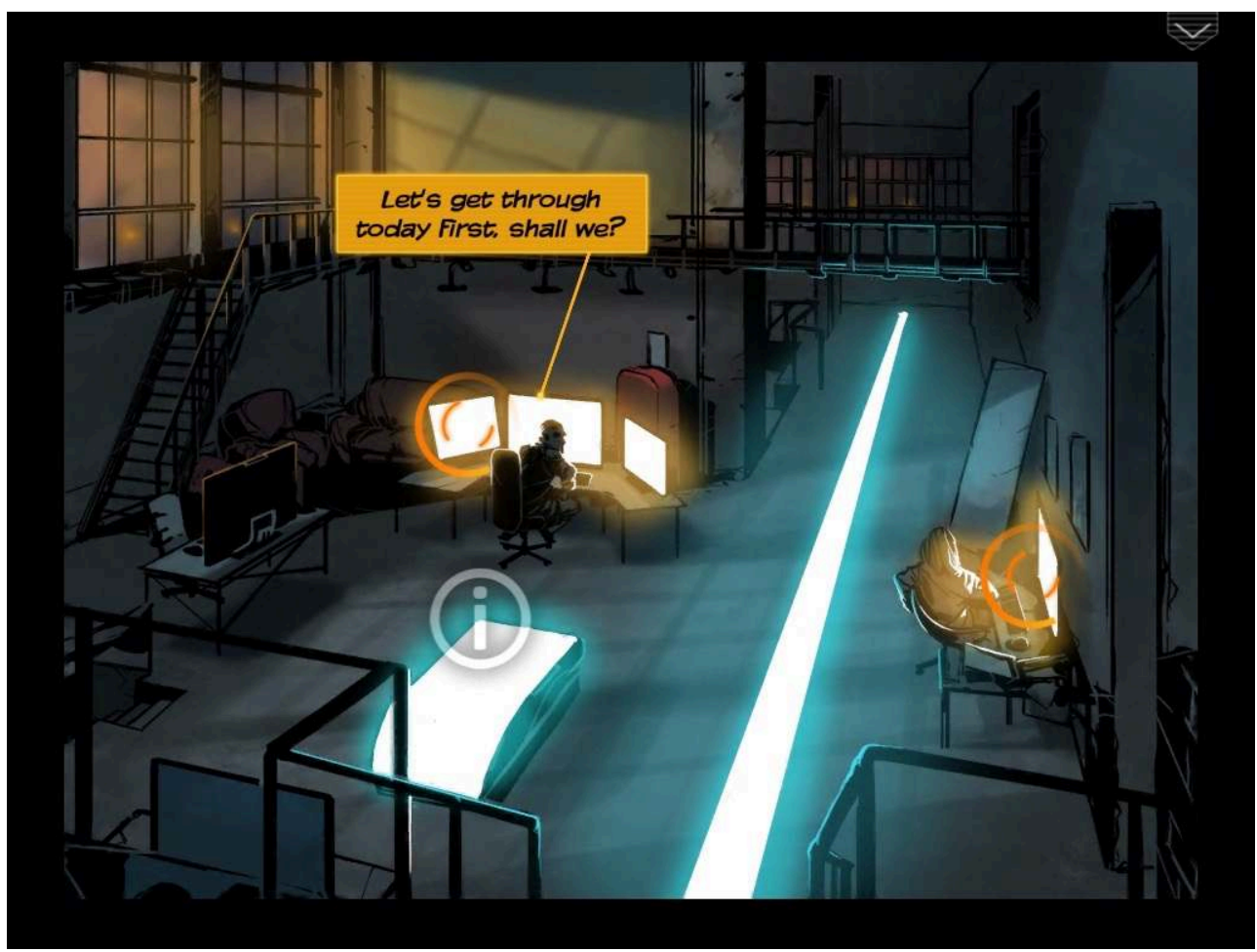

Cette capture d'écran tirée de l'épisode 1 propose par exemple trois points d'interactivité.

Fig 3. Premier hyperlien. Sean Coleman, Felix Mertikat, Verena Klinke et al., 2015, The Butterfly Attack, filmtank $\mathrm{GmbH}$. Application roman graphique.

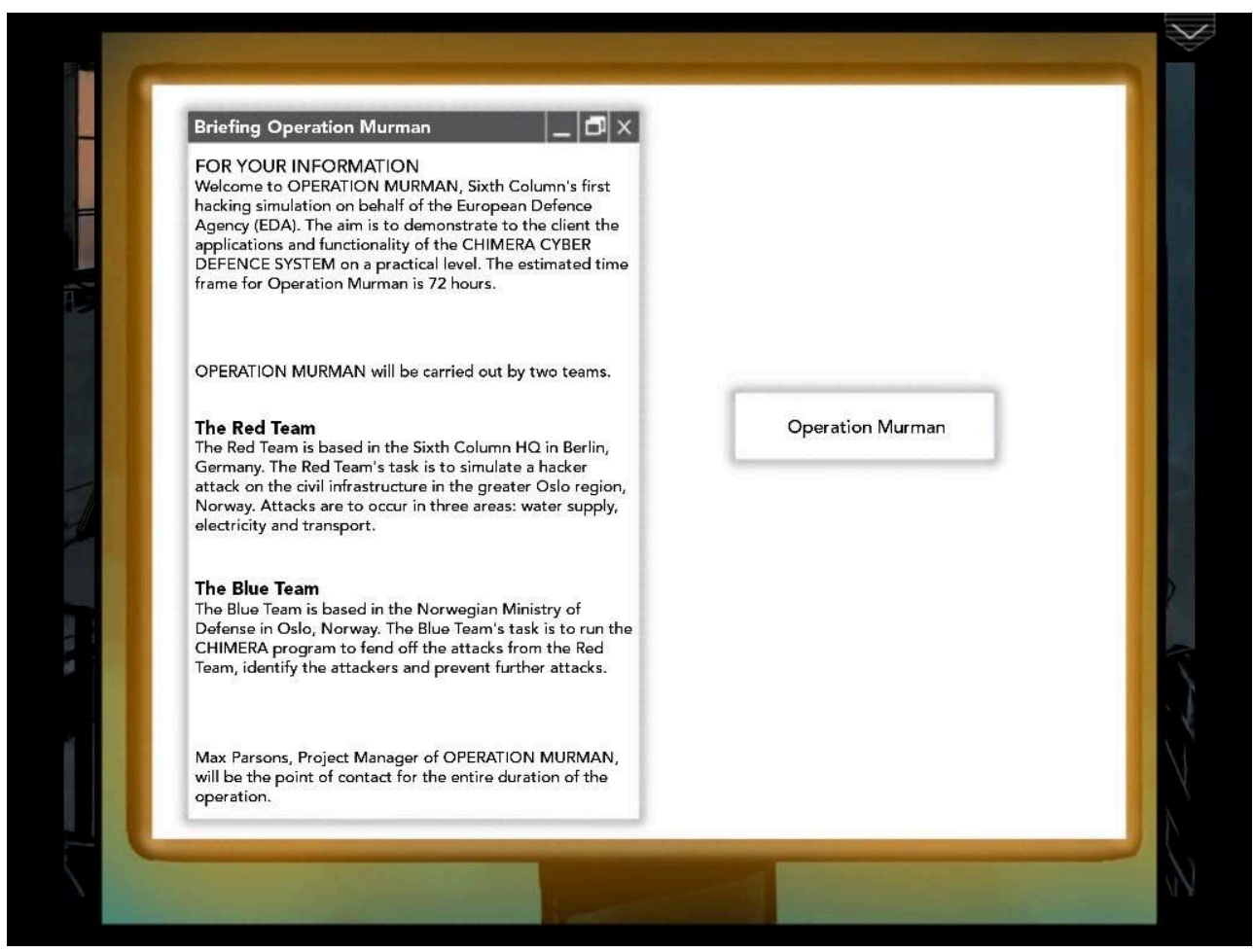


Le premier rond orange présente l'opération de hacking au cœur de la fiction : l'opération Murman organisée par Max et son groupe de hackers dénommé Sixth Column.

Fig. 4. Second hyperlien. Sean Coleman, Felix Mertikat, Verena Klinke et al., 2015, The Butterfly Attack, filmtank GmbH. Application roman graphique.

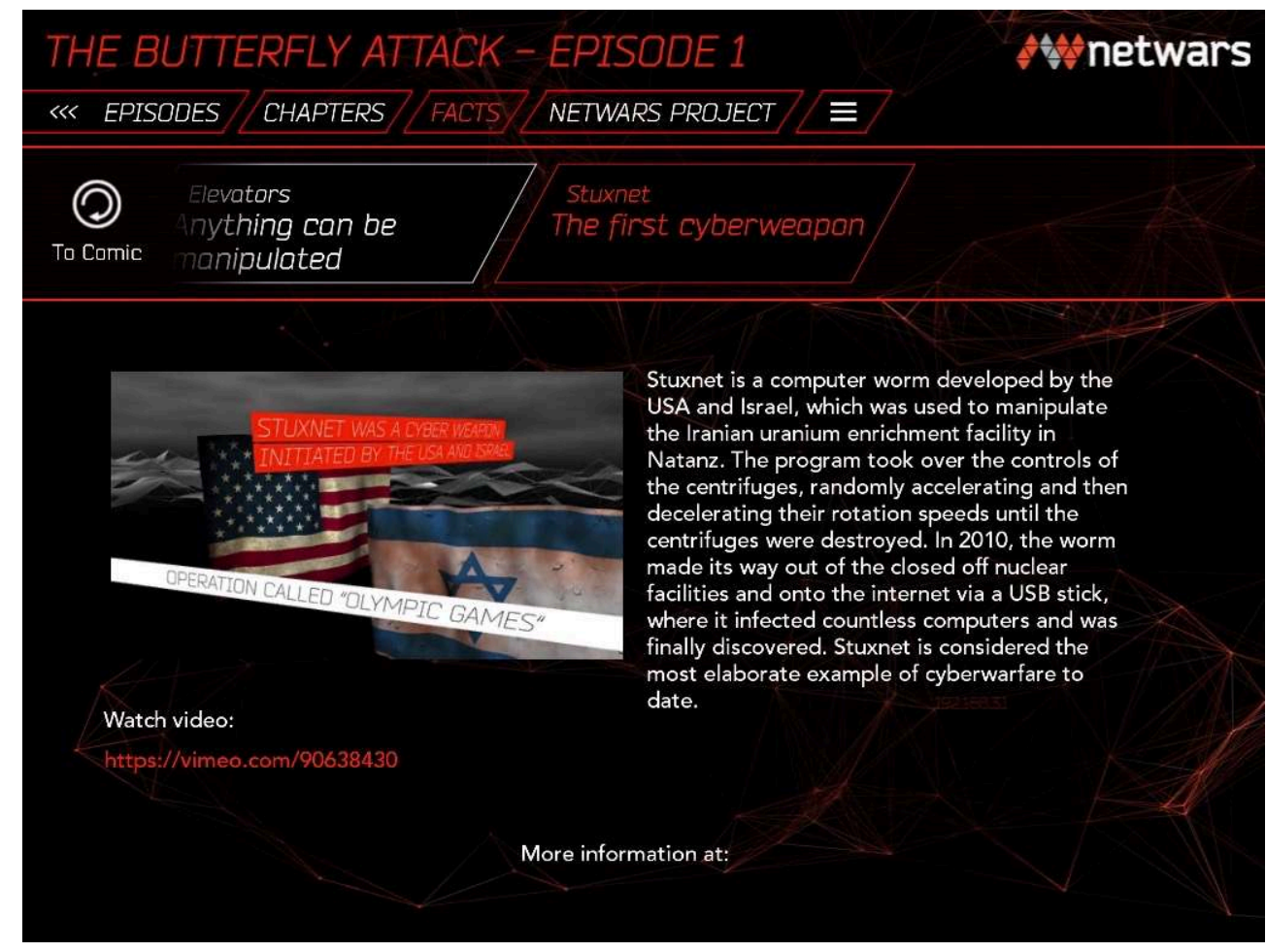

Le second hyperlien quant à lui renvoie à la section « fact » et donne des informations au sujet de l'affaire Stuxnet et de l'opération Olympic Game qui ont résolument inspiré la fiction (Langner 2011) ${ }^{4}$. Un lien vers une vidéo Vimeo est proposé ainsi que vers le site de netwars pour le lecteur qui désirerait en savoir plus. Il est intéressant de noter que les écrans d'information sont illustrés par des vignettes tirées de la fiction. Au sein de l'application, textes et images se font donc écho comme références et récit fictionnel. 
Fig. 5. Troisième hyperlien. Sean Coleman, Felix Mertikat, Verena Klinke et al., 2015, The Butterfly Attack, filmtank GmbH. Application roman graphique.

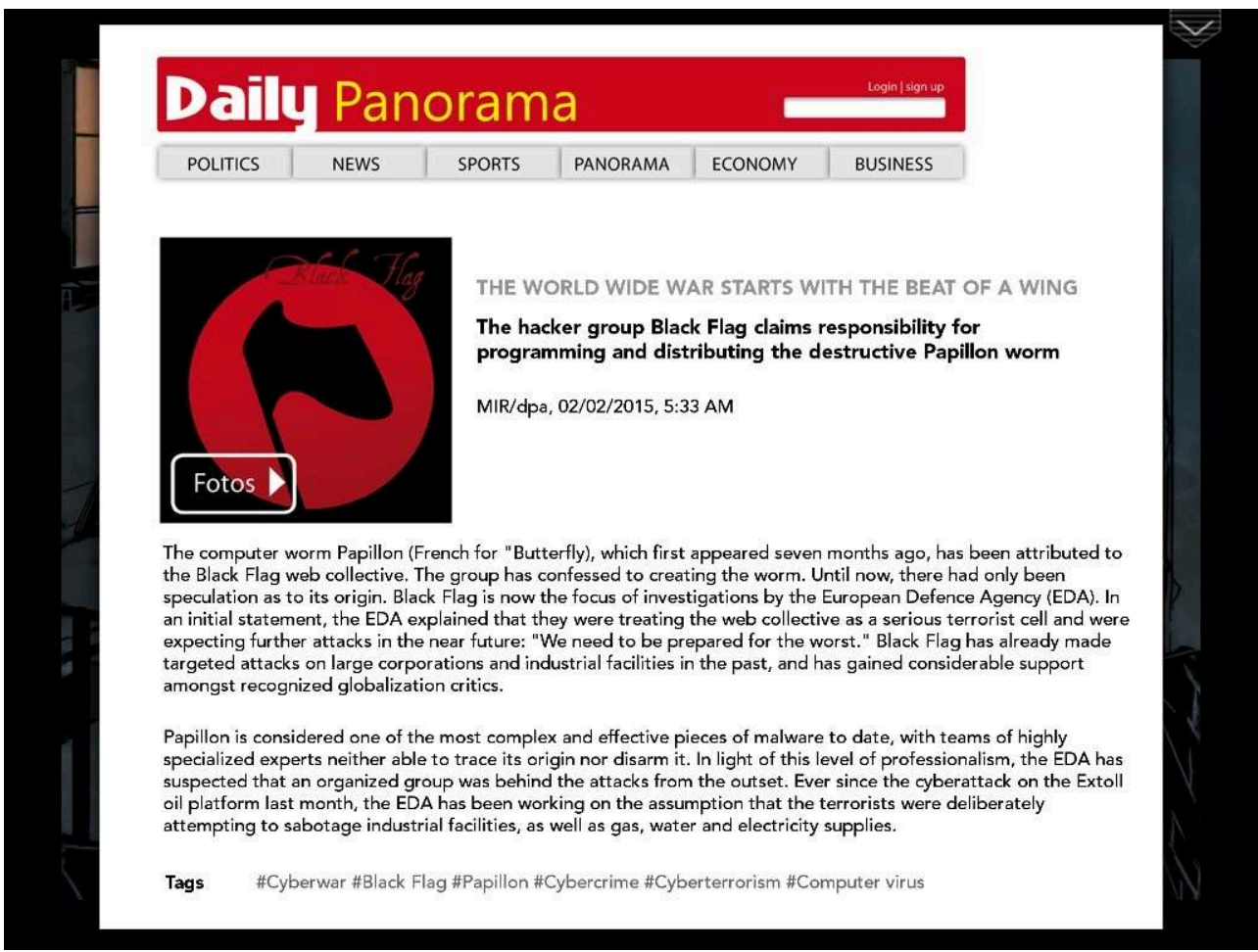

Le troisième point d'interactivité fait basculer de nouveau le spectateur dans l'univers fictionnel de The Butterfly Attack en lui proposant un article de journal sur une attaque du groupe de hackers Black flag et leur « papillon virus » dévastateur.

Néanmoins, dans ces exemples, si les faits inspirent la fiction ou la documentent, ce qui appartient à la fiction reste clairement séparé des données référentielles. Or, au fur et à mesure du parcours transmédia dans netwars, la frontière entre faits et fiction semble être de plus en plus poreuse. C'est sans doute dans le web-documentaire que cette hybridation est la plus prégnante et le personnage du Salesman, narrateur du documentaire et personnage fictionnel des plus cyniques, l'incarne particulièrement bien. Ce dernier n'est jamais nommé dans le web-documentaire mais il peut être identifié grâce à la lecture du roman de Coleman. En effet, dans le récit proposé par l'audiobook, l'e-book et le livre papier, le personnage principal, Strider, a pour mentor un homme qui se fait appeler The Salesman :

Il avait été introduit au Code, dans sa forme première, par un contact qu'il avait rencontré sur l'un des panneaux d'affichage du Deep Web. [...] Il avait été attiré vers lui par un gars, il supposait qu'il s'agissait d'un gars, qui se faisait appeler le Salesman, et les deux avaient commencé à chatter. [...] C'était le Salesman qui lui avait appris qu'une approche fractionnée pour la fuite de données sensibles ne permettait pas de faire tomber le moindre gouvernement ou de demander des comptes sur leurs actions et décisions aux responsables. Le Salesman s'adonnait à des missions bien plus personnelles pour le compte de ses clients, et lentement, en quelques mois, il avait formé Strider à son mode de pensée et transformé un jeune pirate en colère en assassin froid et calculateur. Un professionnel ${ }^{5}$. (Coleman 2014: emplacement 430 et 440 )

Froid, calculateur, autant d'adjectifs qui s'appliquent à Strider comme au narrateur du web-documentaire. Dans sa cinquième partie («L'industrie de la peur »), il déclare : 
Pourquoi je fais tout cela, je vous l'ai dit, l'argent. [...] Je suis juste un gars, il n'y a rien que je puisse faire pour changer les choses. Je suis juste un Salesman. Et vous? Où vous situez-vous dans tout cela mon ami? Je vous ai dit que nous sommes dans le même bateau, non? Que je ne vous laisserai pas tomber. Eh bien, je mentais, merde, vous travaillez en fait pour moi. Vous faites partie du botnet que je vends au plus offrant. Établir et exploiter la confiance, rappelez-vous. Prenez soin de vous maintenant, je vous remercie de votre collaboration, et bonne chance pour la prochaine fois ${ }^{6}$.

Le narrateur se présente ici comme étant un «salesman » et le lecteur des ouvrages de Coleman ne peut que l'identifier comme étant le mentor de Strider. D'autant que l'on retrouve dans l'intervention du narrateur du web-documentaire comme dans la description du Salesman du roman, une même volonté de recruter des hackers, parmi lesquels le spectateur peut compter.

8 Le Salesman fonctionne donc comme un pont entre les différents médias du dispositif et, tel un virus, il vient contaminer le web-documentaire, le faisant alors apparaître de manière flagrante sous le jour d'un docufiction. Selon Yannick Rech et Éric Triquet, chercheurs et enseignants s'intéressant à l'articulation didactique entre fiction et données scientifiques :

Ce genre hybride se caractérise par un couplage étroit entre d'une part une volonté de réalisme et une recherche d'authenticité portées par la composante documentaire et d'autre part par le souci de laisser libre cours à l'imaginaire de la fiction. Dès lors, la visée est double, également - informative et de distraction portant là comme un défi des ambitions aussi louables que délicates à tenir. (2012:

2)

Et le web-documentaire, ou plus largement le numérique, est un lieu propice au développement des docufictions dans leur volonté d'informer et de divertir, dans la mesure où, comme le fait remarquer Samuel Archibald dans «Vraie fiction et faux documents : notes éparses sur l'assassinat de Bill Gates » :

Le maintien d'un flou entre discours factuels et fictionnels, ou entre formes fictives et documentaires, constitue un trait marquant des esthétiques numériques contemporaines. La réception d'œuvres fictionnelles limites s'effectue souvent désormais sur la construction progressive d'un cadre pragmatique plutôt que sur son établissement inaugural. Faisant l'expérience d'une fiction, on peut mettre longtemps à comprendre dans quelle proportion et suivant quelle logique il faut associer les propositions d'un texte à un monde fictionnel et à son propre univers de référence, voire se tromper complètement. (Archibald $2011: 78$ )

\section{La figure du doute?}

Dans netwars, il faut au spectateur un peu de temps et la découverte des différents médias en jeu pour comprendre la logique du dispositif transmédia, la manière dont il jongle entre discours factuels et fictionnels. C'est en regardant le web-documentaire que celui-ci se révèle plutôt docufiction, malgré le cadre pragmatique établi par son péritexte, notamment le site web du projet qui le présente comme un « Interactive web documentary ». Comme le note Françoise Lavocat dans Fait et fiction (2016), l'ère numérique propose des frontières poreuses, mais aussi mouvantes entre fait et fiction. L'univers netwars les reprend à son compte particulièrement parce que cette porosité permet de créer ponctuellement du doute dans l'esprit du spectateur et d'apporter de l'eau au moulin des théories complotistes qui président à ses intrigues. 
10 Ainsi, dès la première intervention du Salesman dans l'épisode 1 , est mis en doute le statut référentiel des faits de hacking, de l'existence d'une cyberguerre et donc de tous les objets $\mathrm{du}$ documentaire: «Est-ce pour de vrai ou s'agit-il d'un canular hollywoodien ${ }^{7}$ ? ", déclare-t-il. En créant d'emblée un doute, il contribue à l'atmosphère paranogène du projet. En parlant de farce hollywoodienne, il interroge la possibilité que le web-documentaire que le spectateur est en train de découvrir appartienne à cette longue lignée de faux documentaires qui aurait été inaugurée par le drame radiophonique The War of the Worlds de Georges Orwell (1938). La légende veut qu'il ait piégé les auditeurs et provoqué des émeutes. Dans un même registre on peut également citer Opération Lune, canular documentaire de William Karel diffusé par Arte le $1^{\mathrm{er}}$ avril 2004. Ce dernier démontre comment les images des premiers pas de l'homme sur la Lune auraient en fait été tournées par Stanley Kubrick et qu'il s'agissait d'un complot du gouvernement américain pour faire croire à la victoire des Américains sur l'URSS dans la course à la Lune.

11 De la même façon, la construction des passages vidéo dans lesquels intervient le personnage du Salesman tend à exacerber les doutes du spectateur et à renforcer la paranoïa ambiante; ils sont une transcription visuelle des émotions que l'univers netwars doit susciter chez le spectateur. Le montage est formé de plans très courts qui s'enchaînent rapidement en même temps que le Salesman parle, la voix crée une continuité entre les différents plans.

Fig. 6. Extraits de séquences vidéo du web-documentaire Lena Thiele, Miiqo Studios UG et al., 2015, netwars, filmtank $\mathrm{GmbH}$. web-documentaire
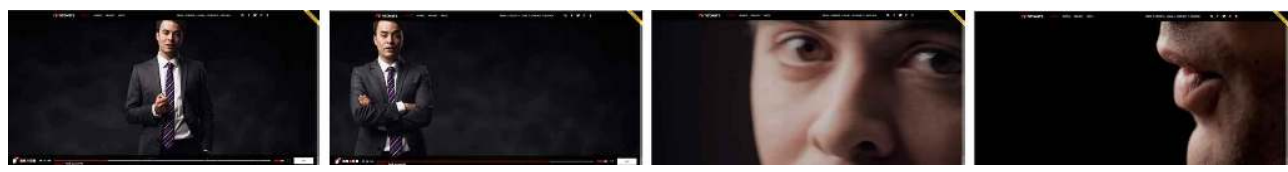

changements de composition qui permettent de voir le personnage une fois droite de l'écran, une fois en haut, en bas, etc., donnent une sensation d'ubiquité : il peut être partout en l'espace d'une milliseconde, de la même façon que notre connexion Internet nous offre une téléprésence (Weissberg 1999). Simultanément, son discours nous conduit à penser qu'il maîtrise ce monde connecté dans lequel il navigue avec aisance, ce qui renforce sa domination sur le spectateur. On remarque également qu'il y a de grands écarts de valeur de plan. Nous pouvons voir le personnage en pied, comme voir un gros plan sur son œil, sa bouche, le quart de son visage : ces plans rapprochés dynamisent le montage, mais illustrent aussi la possibilité de faire un focus sur un point très précis de l'image, de la même façon que le Salesman dit pouvoir voir sur le Web les petits détails de notre vie numérique.

Dans l'univers de netwars, l'information est une arme et nous sommes (bientôt) en guerre. Nous pouvons donc observer dans le montage des images de guerre tirées d'archives vidéo (tranchées, bombe atomique, araignée, etc.). Ces quelques plans d'insert apportent énormément d'intertextes : non seulement il s'agit de symboles forts de notre culture, mais ils injectent dans le discours du Salesman violence, terreur et destruction. Ces inserts sont souvent placés dans le montage de façon à terminer visuellement la phrase sonore qui a été commencée par le Salesman. La présence même de ces phrases terminées en images renforce visuellement l'idée qu'il y a de très nombreux jeux de non-dits dans netwars, qu'il existe un réel décalage entre ce qu'il dit et les images que l'on voit, puis ce qu'il en dit après. Ainsi, le discours de ces séquences 
vidéo s'annonce comme dédramatisant alors qu'en réalité il exacerbe les peurs du spectateur. La guerre, par exemple, est présentée non pas comme une série d'actes horribles qu'il ne faut jamais reproduire, mais comme une inévitable conséquence de la nature humaine qui change de forme à mesure que nos connaissances technologiques évoluent: "La guerre: la success story humaine de l'évolution, c'est ce que nous faisons, c'est dans notre nature comme la musique ou le sexe. » La guerre d'aujourd'hui devient numérique: "Cyber guerre: la menace invisible ${ }^{8}$." Le montage crée une comparaison avec la guerre telle que nos pays l'ont connue, la guerre numérique commence alors à faire vraiment peur. Par ailleurs, ces images issues d'archives permettent d'ancrer les interventions du Salesman dans le factuel pour mieux soustendre une fiction aux allures de réalité.

Les théories du complot ou conspirationnistes, ce " grand envers de la fiction » comme les qualifie Samuel Archibald (2011: 78), sont très présentes dans l'ensemble des éléments du dispositif transmédia de netwars, qui du fait de leur convergence (Jenkins 2006) et de leur présence sur Internet, semblent proposer un terreau fertile à la construction de ces fictions.

Comme beaucoup de sous-cultures ayant émergé des franges de la culture populaire au $\mathrm{xx}^{\mathrm{e}}$ siècle, le conspirationnisme est profondément bouleversé, depuis une quinzaine d'années, par l'avènement d'Internet. Comme les communautés d'admirateurs, les tenants de théories du complot ont trouvé dans le cyberespace un moyen particulièrement efficace de diffusion et de recrutement, à grande échelle et en temps réel. La pensée conspirationniste participe en ce sens d'une culture de la convergence telle que l'a décrite Henry Jenkins. (Archibald 2011 : 80)

Le discours du Salesman, grand recruteur, apparait dans cette droite ligne, mais il faut également noter que le groupe de hackers, héros du roman graphique, se dénomme "The 6th column", faisant écho à "la cinquième colonne ». Ce mythe politique qui aura largement nourri les imaginaires complotistes et dont les origines datent de la guerre civile espagnole de 1936-1939, sera popularisé par les journaux dans les années 1940 en regard de la défaite de la France. L'expression "cinquième colonne » désigne tout ennemi intérieur, traîtres infiltrés au sein d'un État, d'une armée ou d'une organisation, prêt à effectuer un travail de sape ou à se réveiller pour prendre à revers lors d'une attaque extérieure. Dans le roman graphique, la sixième colonne effectue une simulation d'attaque (l'opération Murman) pour prouver aux militaires la qualité de leur système de cyber-protection : CHIMERA. Pour les besoins de la démonstration, les hackers sont divisés en deux équipes. La première équipe incarne le camp des cybercriminels en charge de mettre à mal le système hydraulique, d'énergie et de transport d'Oslo, la seconde est en charge de le défendre. Or ce qui ne devait être qu'une simulation, s'avère finalement avoir des répercussions sur la ville norvégienne qui se trouve subitement privée d'énergie : les pirates ont été piratés par Nightshade, un hacker malfaisant. 
Fig. 7. Sean Coleman, Felix Mertikat, Verena Klinke et al., 2015, The Butterfly Attack, filmtank GmbH. Application roman graphique

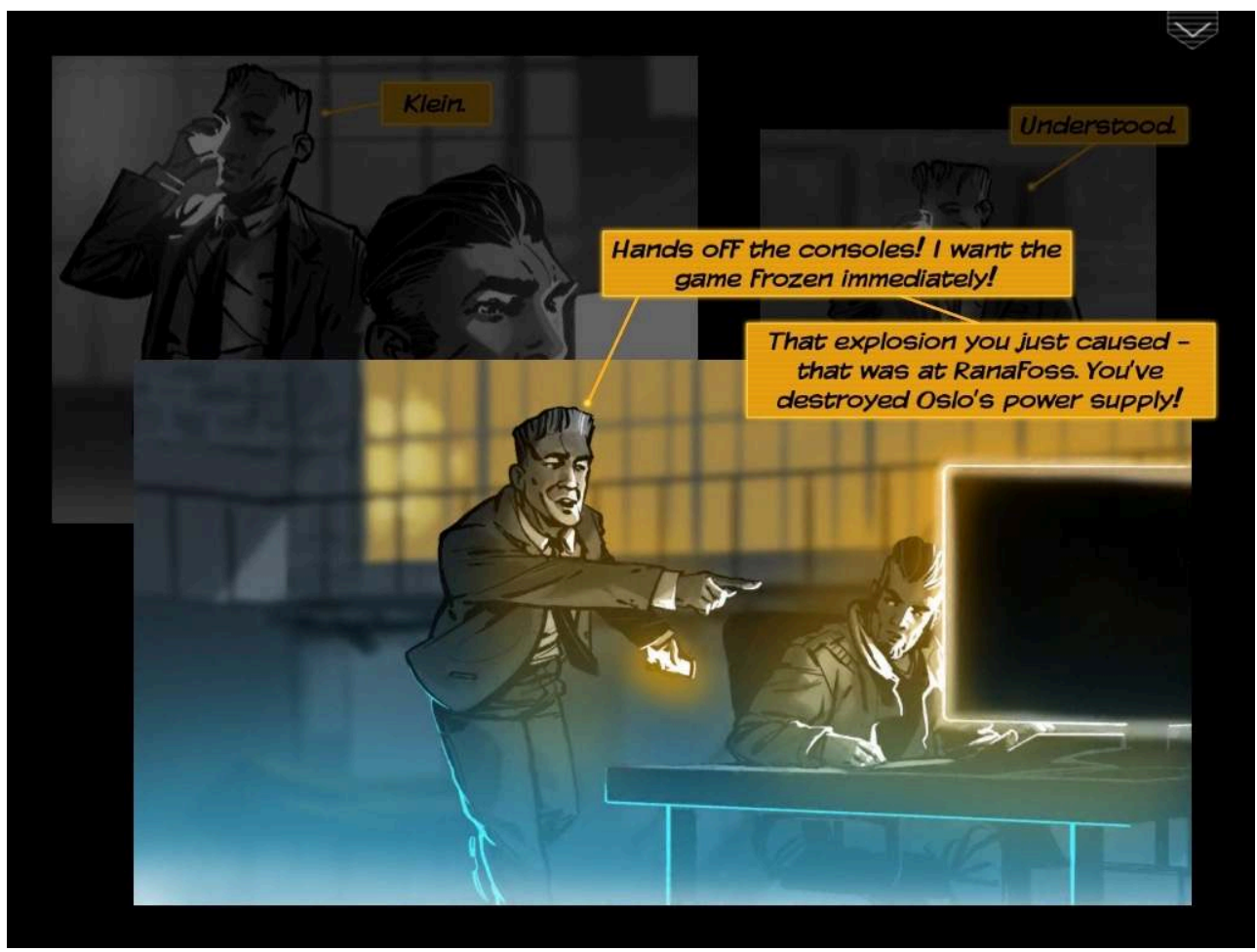

Ce qui, dans la diégèse, ne devait être qu'une projection fictive s'accomplit finalement. De même, la menace de la cyberguerre selon l'univers netwars n'appartient plus à l'ordre de la science-fiction et le dispositif transmédia netwars, dans sa dimension métaleptique, est capable d'avoir des répercussions et de pénétrer le monde du spectateur et ses outils. Ainsi, l'attaque de hackers appartient à tous les niveaux diégétique et spectatoriel, nourrissant de ce fait la paranoïa ambiante.

\section{Le spectateur, paranoïaque?}

Dans l'univers de netwars, le spectateur est invité à se prendre au jeu de la confusion entre faits et fiction, à se laisser griser par l'ambiguïté de ce qui relève de l'un ou de l'autre. Ce projet répond à cette tendance humaine pointée par Jean-Marie Schaeffer dans Pourquoi la fiction?: "[...] nous recherchons activement les simulacres de toute sorte et rien ne nous plaît autant que de nous (faire) prendre au jeu» (1999 : 22). La structure transmédia de netwars va permettre, grâce aux différents niveaux de réalité de chaque support, d'exacerber l'impression que la fiction déborde de son cadre : elle devient intrusive, invasive, tellement ancrée dans la réalité de notre quotidien qu'on ne sait plus très bien dissocier faits et fiction.

\section{Une fiction intrusive?}

16 Un des premiers éléments permettant de rendre le projet intrusif est l'implication du spectateur et de son empreinte numérique dans la diégèse. Par exemple, dans le webdocumentaire, dès le premier épisode, on peut voir s'afficher rapidement dans une des séquences vidéo du Salesman des données personnelles propres à chaque utilisateur 
(adresse IP, opérateur, pays, ville) en arrière-plan du personnage qui parle : « Tu es déjà impliqué $[. .$.$] tu fais partie intégrante de ce qui va se passer ensuite { }^{9}$. "

Fig. 8. Lena Thiele, Miiqo Studios UG et al., 2015, netwars, filmtank GmbH. web-documentaire

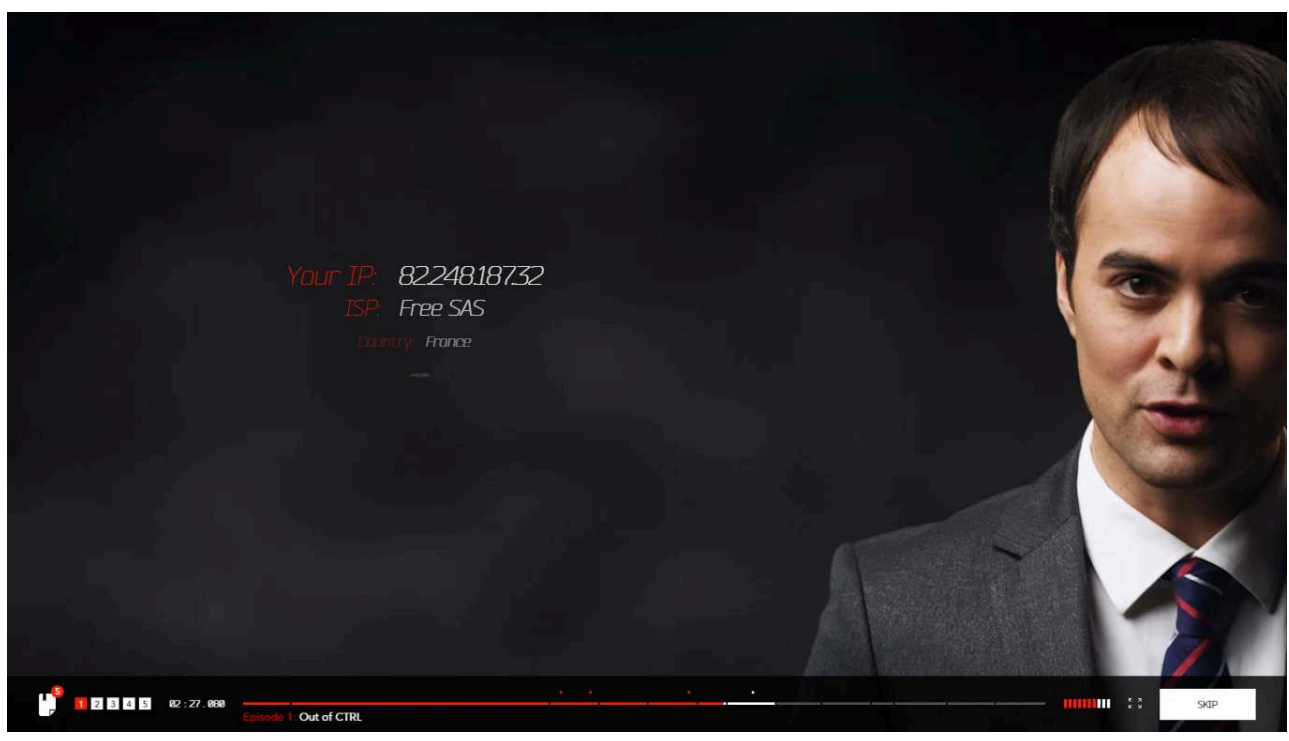

17 Nous sommes ainsi, dès le début, impliqués malgré nous, ce qui entraîne déjà une première sensibilisation sur l'accessibilité et la protection de nos données.

Toujours dans ce même épisode s'affiche une carte représentant les cyberattaques entre 2012 et 2014 qui proviennent d'une source attestée et citée par hyperlien : http:// www.sicherheitstacho.eu/?lang=en. D’une manière générale, dans netwars, les hyperliens servent à référencer, attester, et démontrer la véracité des faits. S'ajoute à cette carte le lieu de résidence de l'utilisateur qui est géolocalisé et représenté par une icône unique. La superposition d'informations personnelles et factuelles avec des données représentant des attaques virtuelles permet de créer un fort effet d'immersion du spectateur et le place au cœur du dispositif transmédia comme des cyberattaques. Ces informations factuelles générales et personnelles affichées sur le même plan tendent également à renforcer la paranoïa puisque des données personnelles sont révélées à notre insu. De plus, le personnage du Salesman conclut cette cartographie par « Voyons à quel point vous êtes réellement vulnérables ${ }^{10}$ ", rappelant ainsi que dans netwars l'information est une arme et que la vulnérabilité de nos données personnelles nous met en danger. S'ensuit un quiz permettant à l'utilisateur de tester ses cyberaptitudes, au cours duquel il se rendra compte que chaque clic de réponse aux questions a déclenché un téléchargement inopiné. Le spectateur découvre donc à ses dépens les possibilités d'un piratage de sa navigation. À la façon d'un virus, il découvre sur son ordinateur, dans ses téléchargements, plusieurs fichiers (.zip, .txt, .dmg, etc.) dont les titres enchaînés donnent : « You never know who is in control ${ }^{11}$. » 
Fig. 9. Capture d'écran d'un spectateur du web-documentaire

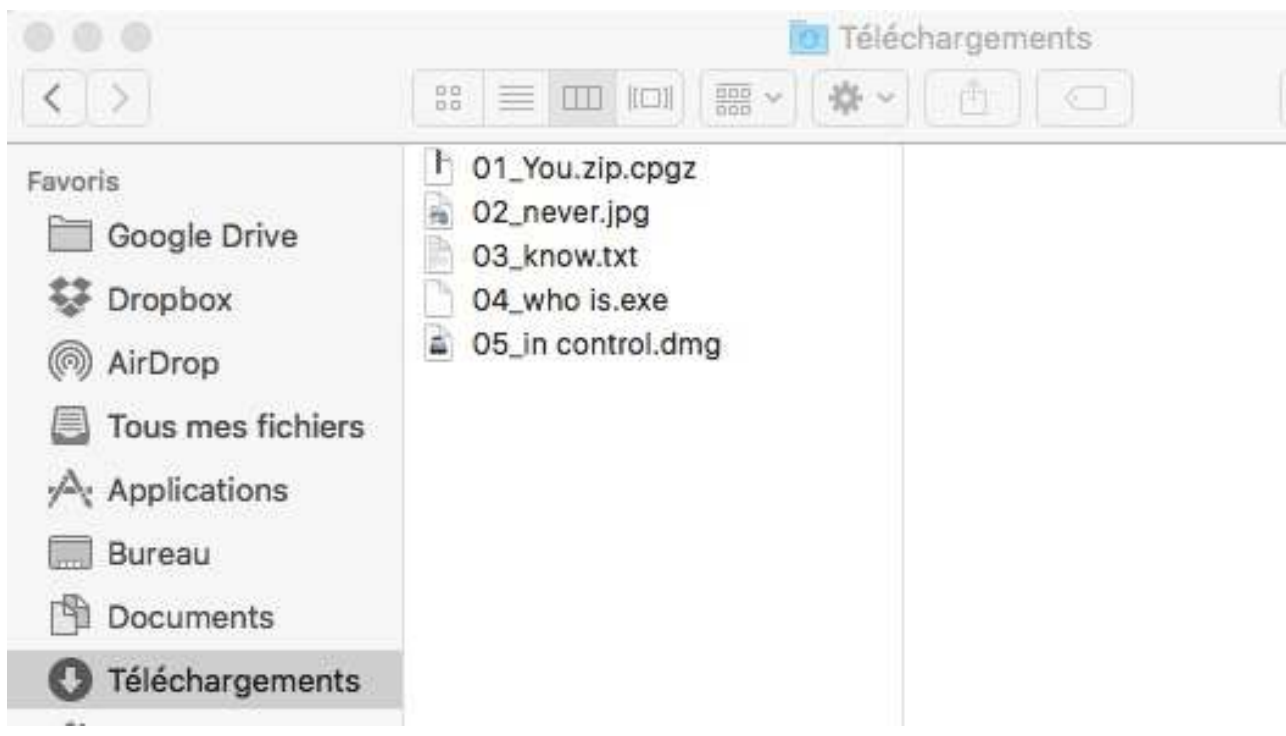

Pour conclure ce piratage, un bandeau déroulant en bas de l'écran affiche un message d'alerte en rouge sur fond blanc (à la manière de ceux des chaînes d'info TV) : «+++ Vous avez pris le risque de nous donner accès à toutes vos données via un téléchargement inopiné +++. Pas si calé, en ce qui concerne la cyber-sécurité. +++ À propos, merci de nous avoir révélé vos forces et vos faiblesses +++ Ne nous appelez pas, on s'en chargera ${ }^{12+++}$.

\section{Se forger son propre avis ou être manipulé ?}

19 Tout au long de la découverte du projet netwars sur les différents supports, le spectateur est conduit à se forger sa propre opinion en naviguant sur le Web vers de très nombreux liens fournis par ses créateurs. Grâce à ces liens, mais aussi à la navigation entre les différents éléments du transmédia, l'utilisateur est incité à élaborer son propre récit en parallèle de ce que lui offre directement netwars. Ce potentiel de proactivité offert au spectateur est assez courant dans les transmédias sans média maître. Si le public doit faire des recherches personnelles qui peuvent l'aider à mieux comprendre ce dont parlent les héros ou figures de l'univers qu'il consomme, alors il se sentira plus impliqué, pourra rentrer dans la partie et devenir un héros aux côtés des héros. Cette implication du spectateur est aussi renforcée par la façon dont le Salesman s'adresse au spectateur dans le web-documentaire: "Ami », "Mon ami », "puisque nous sommes amis ${ }^{13}$ ", etc. Cependant, malgré cette sympathie apparente, le Salesman apparaît comme un personnage manipulateur, il remet continuellement en question tout ce qui a été dit précédemment dans la partie plus documentaire : «Des experts, qui fait d'eux des experts ? [...] Il est aussi conseillé que tu te fasses ton propre avis $^{14}$. Il insuffle le doute, puis finit par donner des exemples fictionnels extrapolés à partir de ce que viennent de dire les experts, ce qui a pour conséquence, non seulement de confirmer la parole des experts, mais de faire également naître la paranoïa chez le spectateur:

Si tu ne paies pas pour le produit, tu es le produit, tout le monde sait ça. Bien sûr avec toutes ces informations qu'ils ont sur toi, tu cours le risque d'être éjecté de ce beau monde flambant neuf. En étant empêché d'acheter cette nouvelle voiture, par exemple, parce que tu as fait un excès de vitesse une fois de trop, ou abandonné par 
le service de soins à domicile parce que ta mère a été malade une fois de trop, ou jeté en prison parce que le collecteur d'impôts sait... ${ }^{15}$

Pour finir, le spectateur apprend qu'il a été dupé par ce personnage et qu'il fait luimême partie d'un groupe de piratage que le Salesman a vendu au plus offrant. Cette dernière révélation invite à la responsabilisation du spectateur : il a fait partie du complot malgré lui et s'est fait piéger par le pacte de confiance du Salesman et aussi, peut-être, par celui de la fiction.

\section{Une illusion du choix?}

netwars, dans sa dimension interactive, n'offre le plus souvent au spectateur qu'une illusion de choix: ce qui renforce la domination du spectateur par le sujet et son sentiment d'impuissance, lesquels alimenteront encore une fois la paranoïa ambiante. Par exemple, dans le web-documentaire, épisode 1, un choix s'offre à l'internaute: «Choquez-moi avec des faits» (pilule rouge) ou « laissez-moi dans l'ignorance » (pilule bleue). Il s'agit de noter également que les pilules sont une référence intertextuelle à Matrix qui renvoie, à l'image de netwars, à tout un environnement cybernétique complotiste, en même temps qu'au thème de la confusion entre rêve et réalité. De plus, dans le film des Wachowski, la scène des pilules fait explicitement référence à Alice au pays des merveilles ${ }^{16}$, Alice qui confond le plus souvent réalité et fiction. Cependant, dans netwars, si le spectateur décide de cliquer sur « laissez-moi dans l'ignorance ", il accède à une page YouTube dont la vidéo a été supprimée. Face à cette impasse, il ne peut que poursuivre avec la pilule rouge.

Fig. 10. Capture d'écran d'un spectateur du web-documentaire.

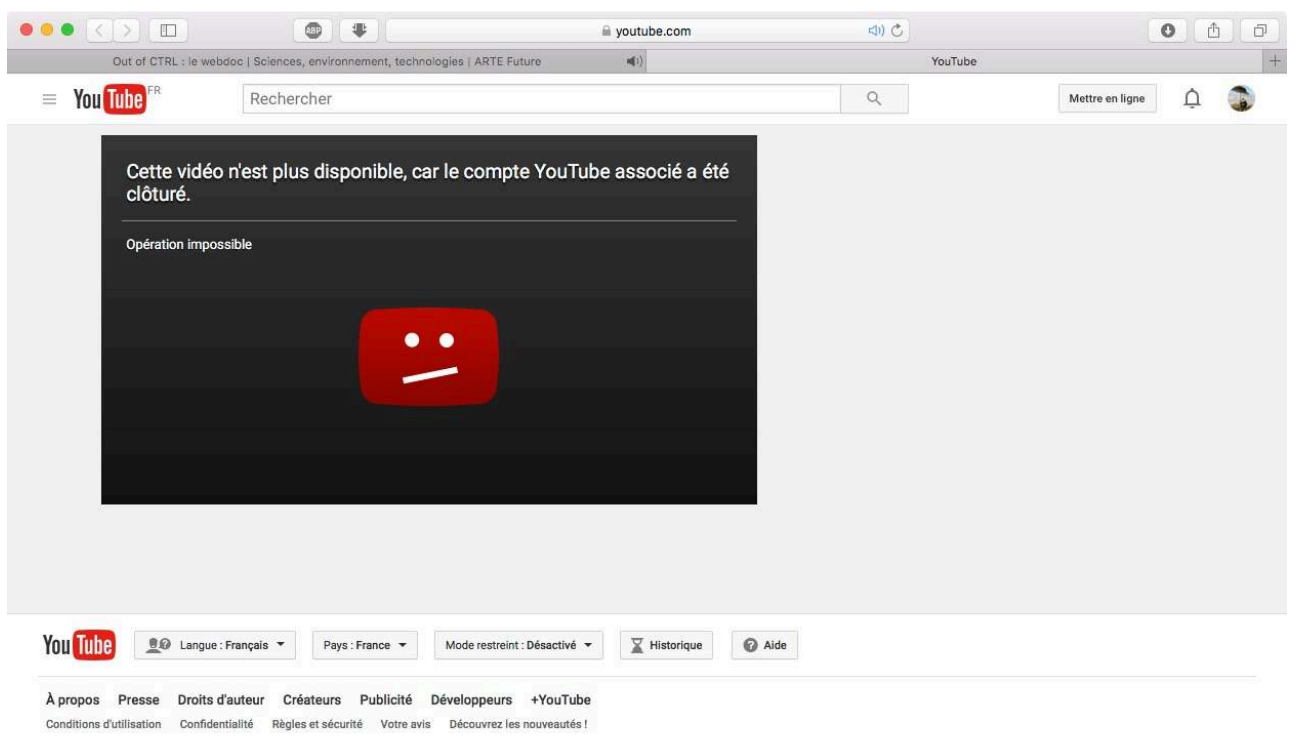

Que les auteurs de netwars l'aient conçu comme cela ou non a peu d'importance, cette présence engendre du doute et démontre la fébrilité interprétative du spectateur: on ne sait plus ce qui appartient à l'œuvre transmédia ou non, ce qui est fait sciemment ou à l'insu des créateurs, ce qui appartient à la fiction ou pas. La paranoïa naît de cette impasse YouTube : est-elle orchestrée par les auteurs ? Est-ce que YouTube a supprimé leur compte? Le cas échéant, pourquoi ? Pour les faire taire? Un complot? Ainsi, fondée ou non, toute cette réflexion nourrit la fiction. On pourrait ajouter également 
que dans le but d'alimenter cette ambiance paranoïaque, les auteurs de netwars durant leurs conférences de presse et autres présentations offrent des brochures possédant un petit autocollant permettant de masquer la webcam des ordinateurs portables, parce que personne ne sait qui a le contrôle.

\section{Dispositif transmédia : in or out of ctrl?}

Comme abordé en introduction, le projet netwars est composé de cinq médias qui possèdent tous une trame narrative propre, mais construisent ensemble un même univers dans lequel le spectateur navigue à son gré. C'est ce qui fait que nous pouvons qualifier ce projet de transmédia tel que défini par Henry Jenkins (2006). Il existe cependant plusieurs types d'univers transmédias correspondant à la définition généraliste de Jenkins. En effet, l'on peut distinguer trois sous-catégories de transmédia qui s'appuient sur la structure narrative des transmédias et sur la place du spectateur au sein de ces univers : les transmédias à média maître inaltérable, les transmédias à média maître altérable, les transmédias sans média maître (Groupierre 2012).

Les deux premières catégories impliquent une trame narrative principale sur un média unique, nommé média maître, ce qui n'est pas le cas de netwars, car chaque média possède sa propre trame narrative principale qui diffère de celle des autres. Il semblerait donc que netwars appartienne à la troisième catégorie: c'est-à-dire un transmédia sans média maître dans lequel « tous les supports s'assemblent comme dans un puzzle pour constituer un univers cohérent et intégré dans le quotidien réel du spectateur. Le spectateur d'un tel transmédia est donc invité à naviguer sur l'ensemble des supports pour saisir l'ensemble de l'univers qu'ils portent » (Groupierre 2012 : 332). Comme analysé précédemment, chacun de ces médias propose à première vue, soit un récit factuel, soit un récit fictionnel. L'application de bande dessinée interactive, le documentaire télévisé, les romans, le web-documentaire (la série est à venir) bâtissent conjointement l'univers de netwars à mi-chemin entre faits et fiction. Ce transmédia ne possède aucun média maître, c'est-à-dire qu'aucun média n'est présenté comme possédant une trame narrative principale de laquelle découlent des médias secondaires qui détaillent ou poursuivent cette trame principale.

\section{Le hacking de la pensée grâce à la structure transmédia ?}

\section{Les parcours transmédias des spectateurs : torsions de la fiction?}

Cette particularité structurale de netwars implique que la navigation du spectateur entre les médias ne soit pas guidée, puisqu'il n'existe pas une seule trame narrative principale, mais cinq. Contrairement au cas d'un transmédia à média maître où le public sera guidé par cette trame narrative principale plus évidente que les autres, dans netwars, chaque spectateur pourra consommer les différents médias dans un ordre qui lui sera propre. C'est une spécificité importante, car chaque support apparaît alors comme un point d'entrée dans l'univers proposé. Ces différentes manières d'accéder à l'univers vont permettre de toucher un public plus large en intéressant, par exemple, à la fois les personnes à la recherche d'un documentaire sérieux comme celles qui veulent se laisser entraîner dans une fiction aux penchants complotistes. Cependant, quel que soit le choix du spectateur, qu'il commence par l'un ou l'autre, il sera conduit à poursuivre l'exploration de l'univers jusqu'à accéder à un espace hybride faits-fiction. 

médias implique des approches de la fiction tout à fait singulières. Par exemple, si une personne décide de commencer netwars par un média présenté comme une fiction telle que les romans ou l'application, pour lui, netwars sera d'abord une fiction que les autres médias viendront documenter et justifier. La fiction semble alors être un récit littéraire non référentiel (Hamburger 1973, Cohn 2001) auquel on ajoute dans un second temps des références. Ce parcours sera donc vécu comme une fiction renforcée par un récit référentiel.

À l'inverse, si une personne découvre l'univers de netwars par un média possédant un registre factuel, comme le documentaire télévisé sur la cybercriminalité, son expérience sera plus proche d'un catalogue de faits attestés qui sont ensuite extrapolés par les autres médias vers une fictionnalité. Cette extrapolation est alors basée sur le principe d'un «imaginez, en théorie ce serait possible ». Ce parcours du factuel vers le fictionnel, semble ainsi conduire le spectateur à l'idée "des mondes possibles » (Leibniz [1765] 1993, Borges 1974, Lewis 1978, Pavel 1988, Guelton 2007). Par ailleurs, s'il commence par un média au pacte fictionnel flou, tel que le web-documentaire, la fiction pourra davantage lui apparaître comme un mélange d'emprunts hétérogènes du réel qui permettent de construire la fiction (Genette 1991). Ainsi, le parcours du spectateur entre les différents médias de l'univers netwars permettra de nuancer son approche de la fiction, et par conséquent modifiera sa manière d'appréhender l'espace flou faits-fictions que fait naître netwars. Par ailleurs, cet espace flou va conduire les spectateurs à partager, échanger et débattre (notamment sur les pages Facebook et Twitter de netwars) à propos de ce qui est faits ou fiction, de ce qui est possible, imaginable ou non. Cela forme une communauté de spectateurs (très fréquemment présente dans les projets transmédias) dont les débats se situent au seuil d'une feintise ludique partagée (Searle 1982, Schaeffer 1999, Kendall 1990), mais à la limite d'une recherche de la vérité.

\section{Le transmédia comme silo?}

De la même façon que l'hyperconnectivité donne l'illusion d'une ouverture, la forme transmédia d'un projet donne l'impression de pouvoir naviguer librement dans un univers qui est en réalité limité pour ne pas dire fermé. En effet, grâce aux nombreux objets connectés et à la démocratisation d'Internet, apparaît un phénomène d'hyperconnexion, une "présence augmentée " (Merzeau 2016) qui semble dans un premier temps offrir les moyens d'une communication démultipliée, des échanges internationaux quotidiens illimités. Cependant, il s'agit de se demander si dans un même mouvement, cette même hyperconnexion ne cantonnerait pas en réalité ses usagers à un mode de pensée sans remise en question qu'ils choisiraient malgré eux, par facilité ou du fait même de la structure des réseaux. Le transmédia pourrait être perçu comme une de ces «bulles de filtres » étudiées par Eli Pariser (2011). Ce concept désigne l'état de l'internaute qui reçoit des informations uniquement filtrées à son insu par les algorithmes de personnalisation qui auront au préalable recueilli et analysé un certain nombre de données le concernant. Ainsi, par exemple, Google personnalise les résultats des requêtes selon les historiques et pratiques de navigation des internautes, enfermant de ce fait le champ de leurs possibles dans ce que l'algorithme devine et projette de leurs desiderata. Les réseaux sociaux procèdent de même en créant des communautés qui se construisent principalement par contiguïté, ressemblances et

Itinéraires, 2016-2 | 2017 
affinités. Les internautes ont tendance à communiquer davantage avec des personnes qui leur ressemblent et ne s'opposent ni à leurs opinions ni à leurs convictions. Le Web fonctionne alors comme un silo:

Les nouvelles ingénieries relationnelles («like», «+1») et les dernières évolutions des moteurs de recherche (personnalisation des résultats) promeuvent un internet des silos, une approche presque carcérale de la navigation, chaque jour plus asservi à la rentabilisation de logiques marchandes d'environnements propriétaires. [...] d'immenses silos documentaires sont apparus (Youtube, Flickr, etc.). (Ertzscheid 2012)

Et Louise Merzeau de compléter :

Puis le web s'est démocratisé et, en se massifiant, son rythme s'est accéléré pour mieux s'uniformiser. Comme on a remplacé les passages obscurs par de larges boulevards et des grands magasins, on a substitué au bric-à-brac de sites aléatoirement reliés les uns aux autres des «murs » où tout est disposé pour que l'utilisateur n'ait plus qu'à cliquer sur des boutons. On ne surfe plus, on « tapote». Les mérites de la déambulation ont dès lors cessé d'être mis en avant : tout doit être accessible en un clic, sans détour, sans errance et sans délai. À cette fin, les grands acteurs du web mettent en place des stratégies de silo, où chaque plateforme circonscrit un univers clos censé se suffire à lui-même, afin que l'internaute n'ait plus la moindre envie de s'évader. Les navigateurs eux-mêmes cèdent le pas aux applications, lesquelles ne communiquent a priori qu'avec des outils de la même firme. Dernière étape de cette mise à mort de la cyberflânerie, l'internet des objets est supposé achever d'orchestrer le monde sur l'unique partition de notre profil calculé. (Merzeau $2014: 21$ )

La toute-puissance et la liberté promises par l'hyperconnexion apparaissent ainsi comme des illusions, fruits d'une bulle de confort intellectuel dans laquelle l'internaute se gargarise de sa propre pensée reflétée par celle des quelques autres, à Hong Kong ou en Amérique, qui pensent comme lui. Une façon d'échanger davantage, mais sans jamais se confronter à ses proches, ses voisins (géographiquement) ou à des inconnus qui auraient une autre vision du monde et de la vie.

De la même façon que le mécanisme illusoire d'une super communication semble davantage être une zone de confort intellectuel, de la même façon que «l'Internet des silos" nous contraint et nous guide, le transmédia se dévoile comme un réseau d'apparence ouvert, mais qui est en réalité fermé, clos sur lui-même. Il contraint le spectateur dans un entre-soi, appelé par une force centrifuge, celle du transmédia. Le spectateur, dans netwars, ne peut pas sortir de l'univers pluri-supports créé pour lui. Il en a l'impression grâce à la navigation entre les différents médias composant netwars, mais il reste dans la zone prévue, il explore les hyperliens fournis, il échange avec la communauté netwars sur les pages créées à cet effet, etc. Ainsi, le projet est conçu de telle manière que le spectateur navigue très aisément d'un support à un autre : les liens narratifs facilitent cette navigation, ainsi que le site web du projet qui présente synthétiquement tous les supports qui le constituent. La transversalité est effective et nous sommes invités à passer à notre gré, du documentaire au livre audio, du roman graphique au web-documentaire, etc. Cependant, cette transversalité n'est patente qu'entre les différents médias de netwars et nous sommes donc face à une liberté illusoire, un silo qui se limite aux médias conçus et réalisés par les auteurs de netwars. Cette interactivité limitée, le mirage de liberté, l'illusion de se faire son propre avis sans aller au-delà des références sélectionnées et fournies tendront à renforcer la paranoïa ambiante que les concepteurs du dispositif ont souhaité mettre en place. Le spectateur 
est confortablement installé dans le silo netwars, le hacking de sa pensée a déjà lieu, mais il ne le sait pas.

\section{Le transmédia, un dispositif de contrôle?}

30 Si nous parlons de "dispositif » pour qualifier le projet transmédia netwars, c'est en embrassant toute la complexité du terme, épousant le sens courant et tout le poids théorique que celui-ci porte, principalement chez Michel Foucault. Le dispositif est, comme on le sait, un terme décisif chez Foucault dans les années 1970 :

Ce que j'essaie de repérer sous ce nom c'est [...] un ensemble résolument hétérogène comportant des discours, des institutions, des aménagements architecturaux, des décisions réglementaires, des lois, des mesures administratives, des énoncés scientifiques, des proportions philosophiques, morales, philanthropiques; bref, du dit aussi bien que du non-dit, voilà les éléments $d u$ dispositif. Le dispositif lui-même, c'est le réseau qu'on établit entre ces éléments [...]. Par dispositif, j'entends une sorte - disons - de formation qui, à un moment donné, a eu pour fonction majeure de répondre à un usage. Le dispositif a donc une fonction stratégique dominante... J'ai dit que le dispositif était de nature essentiellement stratégique, ce qui suppose qu'il s'agit là d'une certaine manipulation de rapports de force, soit pour les développer dans telle direction, soit pour les bloquer, ou pour les stabiliser, les utiliser. Le dispositif, donc, est toujours inscrit dans un jeu de pouvoir, mais toujours lié aussi à un ou des bornes de savoir, qui en naissent, mais tout autant, le conditionnent. C'est ça le dispositif : des stratégies de rapports de force supportant des types de savoir, et supportés par eux. (n.s.) (Foucault $1994: 299)$

Selon le philosophe, le dispositif possède donc une connotation aliénante et négative. $\mathrm{Du}$ «dispositif de surveillance» (Foucault 1975) incarné par le Panopticon, au « dispositif de sexualité » (Foucault 1976), les dispositifs foucaldiens sont les lieux de l'inscription technique d'un projet social total, imposant un contrôle sur les corps et les esprits.

\section{Un panoptique transmédia?}

31 Le panoptique, système carcéral imaginé par les frères Bentham à la fin du XVIII siècle, est au cœur de la construction du concept de dispositif chez Foucault dans sa fonction stratégique et contrôlante. L'objectif du panoptique est de favoriser la surveillance des détenus dont les cellules sont placées en cercle au centre duquel se trouve une tour de contrôle permettant aux gardiens de voir sans être vus. Au sein d'une telle architecture, les détenus se sachant potentiellement surveillés à tout moment sont conduits à effectuer une sorte d'autocensure, d'autosurveillance générée par la crainte même d'être vus, et ce alors que le gardien n'est peut-être pas en poste. Le panoptique repose donc sur sa qualité paranogène. netwars reprend à son compte l'idée du panoptique et propose de voir le Web comme tel : un lieu où il est possible d'être vu à son insu. Et, à ce titre, les hackers capables de pénétrer les ordinateurs de tout un chacun ${ }^{17}$, mais aussi tous les systèmes de surveillance comme la NSA, ainsi que sans doute Google, prendraient la place des gardiens des Bentham dont l'aspect menaçant dépend moins de leur grand nombre que de leur positionnement stratégique et de leur possible présence. Le motif de la surveillance est donc omniprésent au sein des différents éléments transmédias. On le retrouve, par exemple, dans le premier épisode du roman graphique lors des vignettes montrant le meurtre des parents du héros, Max. 
Fig. 11. Sean Coleman, Felix Mertikat, Verena Klinke et al., 2015, The Butterfly Attack, filmtank $\mathrm{GmbH}$. Application roman graphique.

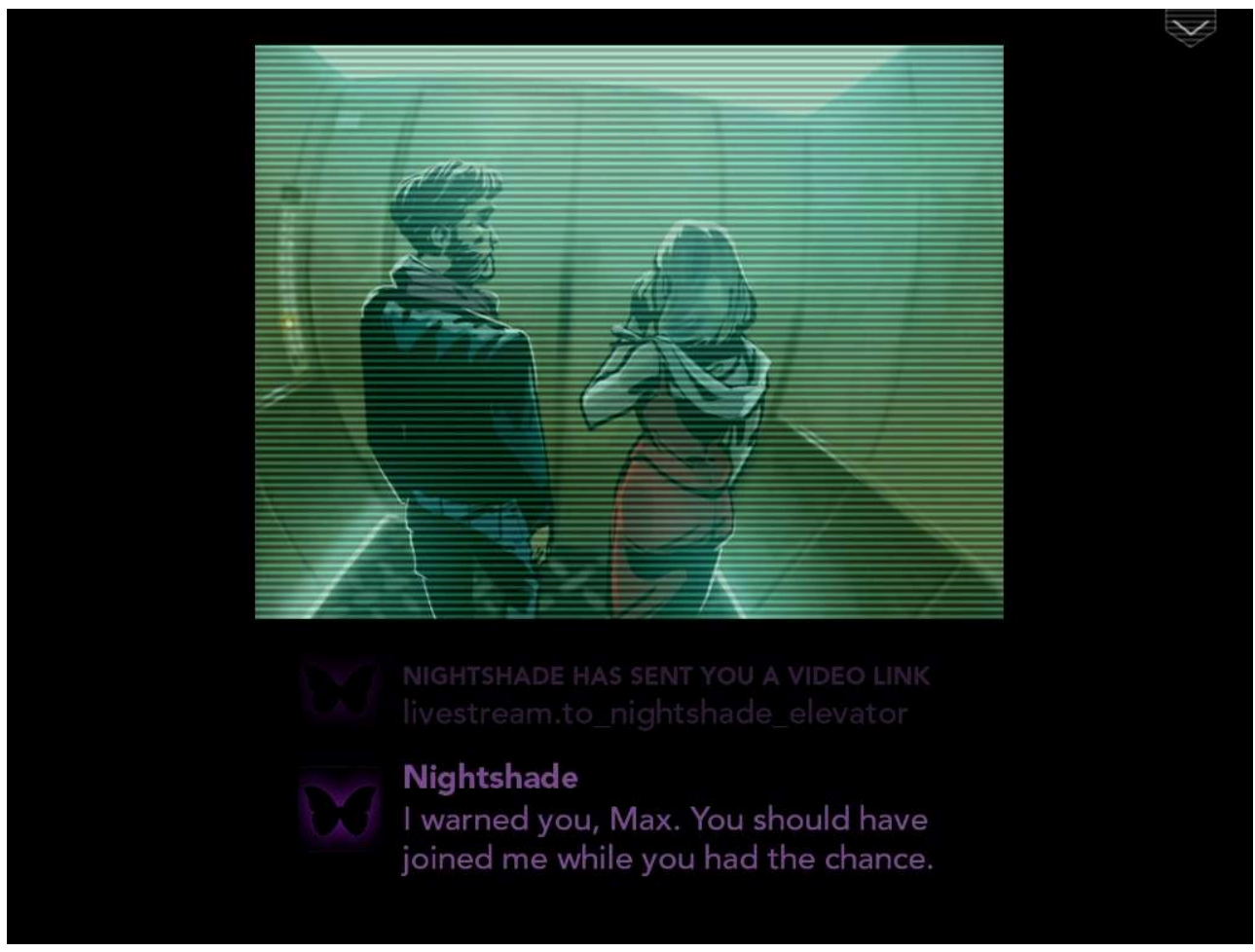

32 Grâce à une image stylisée, imitant les captures de caméra de surveillance, on observe le couple dans l'ascenseur qui sera piraté par le dénommé Nightshade : cette ombre dans la nuit, corroborant l'idée de la menace invisible. On retrouve un même effet CCTV dans le documentaire télévisé. Lors de l'introduction des différents experts intervenant, l'image passe en noir et blanc et de petites lignes horizontales apparaissent, puis un insert permet de l'identifier.

Fig. 12. Michael Grotenhoff, 2015, netwars, filmtank GmbH. Documentaire TV.

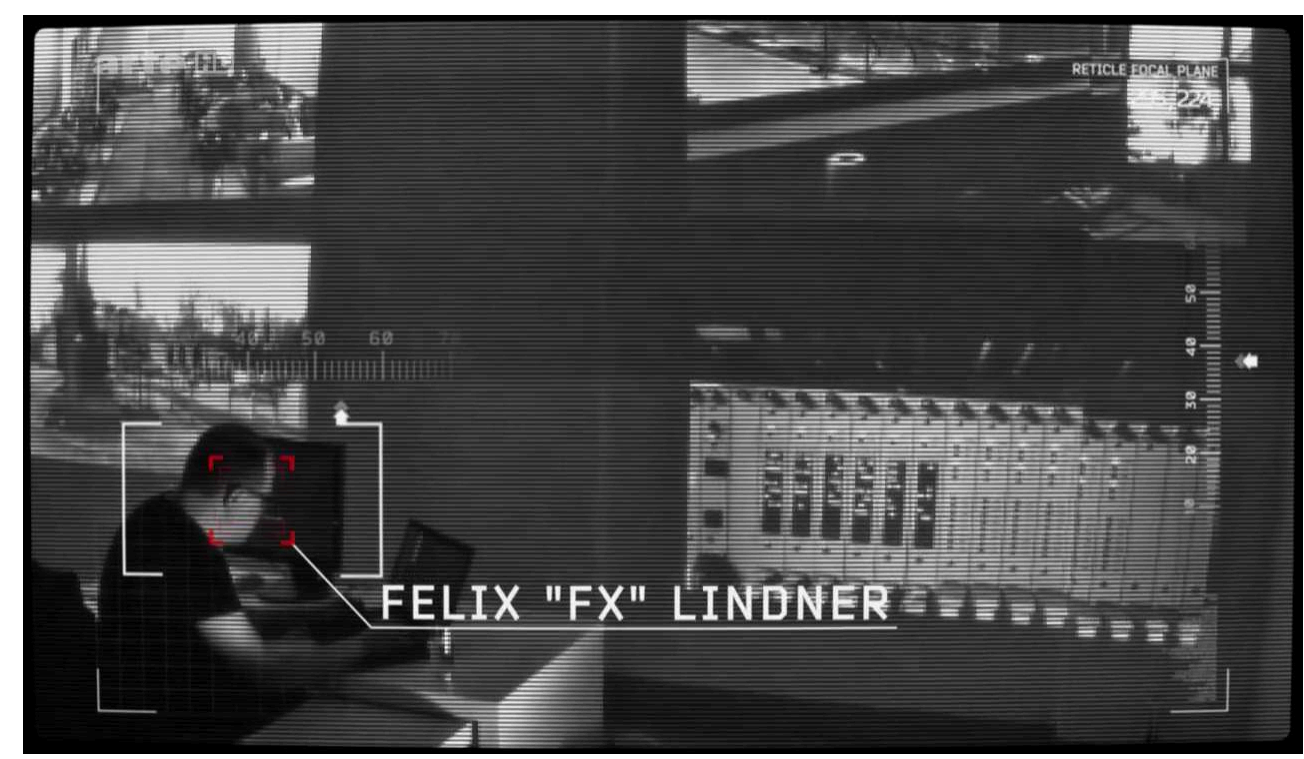



s un même registre, la scène de simulation d'une cyberattaque est filmée en travelling latéral, au cœur de la nuit, montrant au second plan, baigné d'une lumière bleutée froide, des fenêtres de bureaux, alors que la caméra semble être située dans l'immeuble d'en face, à demi cachée.

Fig. 13. Michael Grotenhoff, 2015, netwars, filmtank GmbH. Documentaire TV.

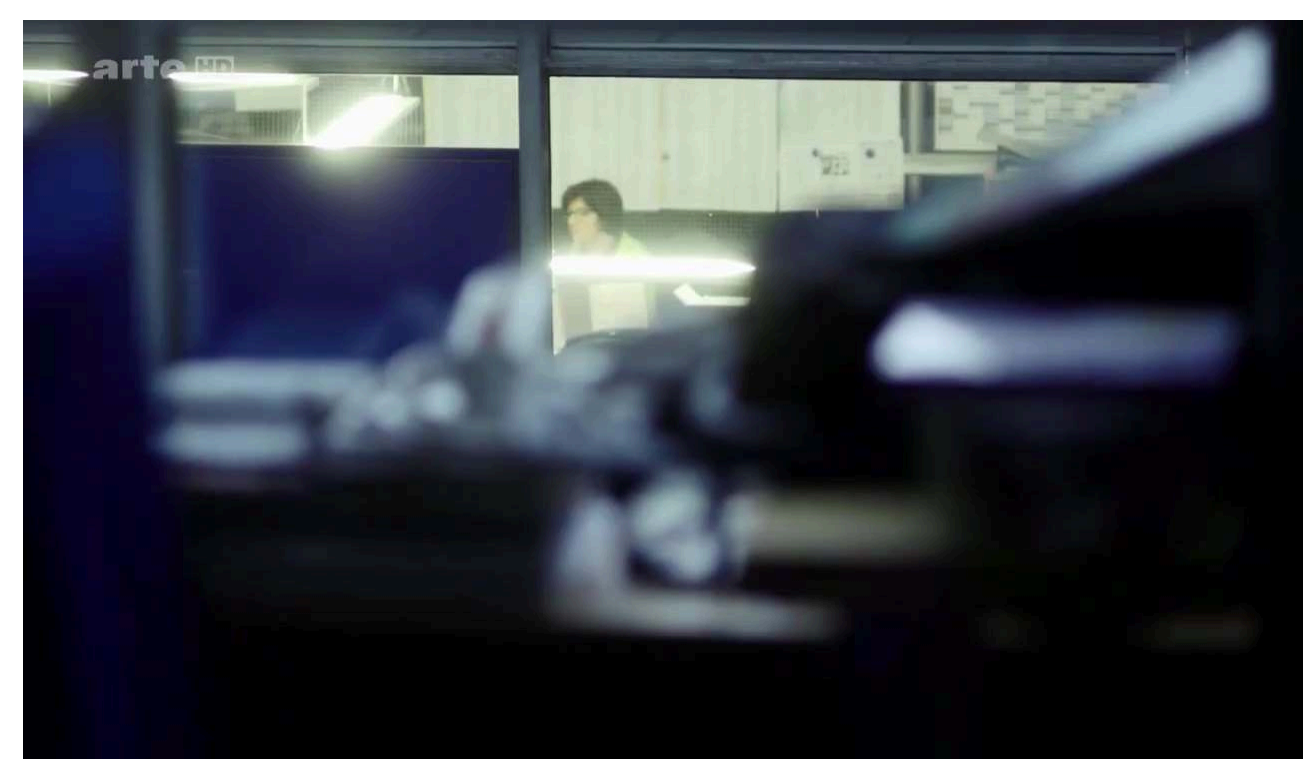

On entend alors en voix-off Frank Boldewin, expert en sécurité informatique, dire: "C'est un peu comme si tu t'introduisais dans un immeuble de bureau. » La caméra subjective, ajoutée à l'adresse au spectateur : «tu», le font entrer dans une posture d'espion, l'entraînant dans la tour centrale du panoptique pour mieux lui démontrer, à l'instar de ce qu'explique l'un des spécialistes convoqués dans netwars, Gabi Siboni ${ }^{18}$, que plus rien n'est secret, qu'il n'y a plus de vie privée. Le roman de Coleman enfonce le clou avec le personnage de Strider, ce hacker qui déclare :

Vous ne me connaîtrez jamais, mais je serai votre arrêt de mort. J'ai vu ce que vous avez fait, je vois tout ce que vous faites. J'ai entendu ce que vous avez dit, je connais vos secrets. Je sais où vous êtes allé, je vous suis partout. Vous n'êtes pas au-dessus de mon examen, je scrute tout ${ }^{19}$. (Coleman 2014 : emplacement 21)

Dans l'univers netwars, être « out of ctrl » c'est donc avant tout subir la menace de voir ses secrets ou plus généralement ses informations personnelles dévoilées. Et comme nous l'avons déjà vu, notamment à l'aune du Salesman du web-documentaire, le spectateur n'est pas à l'abri.

netwars tend à révéler le grand paradoxe des systèmes informatiques connectés contemporains : ils sont le lieu où se noue et se déjoue la surveillance. Le hacker peut $\mathrm{y}$ voir sans être vu, et tout un chacun peut y être observé : il suffit de se référer à l'affaire Snowden et au programme de surveillance électronique de la NSA, ou plus banalement à Google et autres sociétés d'analyse comme Netuitive, qui enregistrent et interprètent le moindre de nos clics sur le Web afin de faire du profilage d'usager et de recueillir un maximum de données (lesquelles seront ensuite vendues à des fins commerciales). 


\section{Le transmédia, « un ensemble résolument hétérogène » ?}

Chez Foucault, on retrouve le dispositif dans la totalité du champ social: pouvoir, savoir, vérité, famille, sexualité, sécurité, école, armée, santé, etc. Le philosophe, dans Surveiller et punir, fait du panoptique le modèle du contrôle social tel qu'il peut s'élaborer dans les sociétés disciplinaires, mais, ainsi que le note Deleuze, il élargit également le concept en proposant le terme de panoptisme afin de décrire tous les systèmes de contrôle quels qu'ils soient.

Quand Foucault définit le Panoptisme, tantôt il le détermine concrètement comme un agencement optique ou lumineux qui caractérise la prison, tantôt il le détermine abstraitement comme une machine qui non seulement s'applique à une matière visible en général (atelier, caserne, école, hôpital autant que prison), mais aussi traverse en général toutes les fonctions énonçables. La formule abstraite du Panoptisme n'est plus "voir sans être vu», mais «imposer une conduite quelconque à une multiplicité humaine quelconque ». (Deleuze [1986] $2004: 41$ )

Chez Foucault, le dispositif est à la fois la manière dont sont liés des éléments entre eux et l'ensemble de ces éléments reliés, ce qui correspond précisément à la description du transmédia. D'autant que Foucault concluait en 1977 dans un entretien pour la revue Ornicar, sur une définition du dispositif comme "ensemble résolument hétérogène ": "Le dispositif lui-même c'est le réseau qu'on peut établir entre ces éléments" (Foucault 1994b : 299). Le Salesman par ailleurs ne s'y trompe pas quand il explique dans le web-documentaire :

Nous sommes tous pris dans la toile de cette chose merveilleusement séduisante et en constante évolution que nous appelons l'interconnexion. Internet a finalement réussi à nous faire comprendre ce que le bouddhisme a essayé de nous transmettre depuis plus de 2000 ans : nous sommes un, nous sommes tous connectés ${ }^{20}$.

On retrouve dans cette citation le réseau dont parle Foucault, l'évocation de ce qui est en même temps singulier et pluriel (Nous sommes un), toujours connecté (nous sommes tous connectés), dans un système sans cesse mouvant et qui, dans son aspect séduisant, recèle des enjeux qui le dépassent largement. Par ailleurs, ce "nous", énoncé par le Salesman, peut désigner non seulement de manière large les internautes, mais aussi les spectateurs de netwars, tous pris dans un dispositif de contrôle.

Ainsi, le transmédia fait donc dispositif. Ce concept, à l'instar de ce que remarque Ivanne Rialland, peut donc, dans le cadre du transmédia, être employé dans sa dimension méthodologique, qui permet d'étudier des assemblages sémiotiquement, médiatiquement et symboliquement hybrides :

Garder au dispositif son sens foucaldien d'un agencement d'éléments hétérogènes réunis stratégiquement et de façon précaire en vue d'un objectif garantit à la notion sa puissance descriptive, qui lui permet de rendre compte des objets sémiotiquement hétérogènes sans leur faire perdre leur unité, ou de penser ensemble le résultat qu'est l'œuvre, le processus technique lui ayant donné naissance, le discours l'accompagnant, sa situation et son devenir. (Rialland 2008)

Cette analyse du dispositif rejoint la définition, devenue canonique du transmédia selon Jenkins :

Une histoire transmédia se déploie à travers plusieurs plateformes médiatiques, où chaque nouveau texte contribue de manière distincte et importante à l'ensemble. Dans la forme idéale du récit transmédia, chaque média réalise ce qu'il fait de mieux, de façon qu'une histoire puisse être introduite dans un film, étendue à la télévision, aux romans, et aux bandes dessinées ; son monde pouvant être exploré à travers le jeu ou expérimenté dans des parcs d'attractions ${ }^{21}$. (Jenkins 1997 : 95-96) 
Entre dépendance et indépendance des unités médiatiques les unes envers les autres, le transmédia se fait résolument dispositif de contrôle dans la manière dont sont disposés stratégiquement ses éléments hétérogènes dans l'objectif de créer un récit. netwars, en embrassant pleinement la définition du dispositif de contrôle foucaldien, du fait des thématiques abordées, de son esthétique, ainsi que de sa structure médiatique et narrative spécifiquement paranogène, révèle de manière plus manifeste que d'autres, cette caractéristique ontologique du transmédia. Les projets transmédias sont des textes, en tant que pratiques signifiantes (Kristeva 1969). Par conséquent, ils impliquent des effets prédéterminés (Iser 1985) que le lecteur est en charge d'actualiser (Iser 1985, Eco 1979). Si comme tout texte, les transmédias font dispositif, force est de constater que netwars va un cran plus loin en contrôlant, voire minorant sciemment le rôle du lecteur. Les effets de lecture dans netwars vont parfois, on l'a vu, à l'encontre de la liberté du lecteur, faisant du transmédia un texte lisible, c'est-à-dire au nombre d'interprétations (extrêmement) limitées et accessibles sans effort, plutôt que scriptible, soit favorisant une pluralité d'interprétations (Barthes 1970 : 10). Ceci étant, ultimement, cet aspect « carcéral » du dispositif n'empêche pas toute liberté de penser ou d'agir, bien au contraire, puisque netwars, dans ses dimensions documentaire et didactique, se veut prise de conscience et appel à la prudence et à l'action : tous les cyber-citoyens que nous sommes doivent impérativement se prémunir de cette prise de contrôle.

\section{Out of Ctrl ?}

40 Être dans ou hors du dispositif, sortir ou non du silo transmédia, donner ou pas ses informations sur Internet, être in ou out of ctrl. Le sous-titre du projet « out of Ctrl » le rappelle bien en utilisant le diminutif propre à la touche de nos claviers, il insiste sur le fait que l'informatique est désormais un enjeu névralgique, voire vital, pour la conservation de notre liberté et de notre libre arbitre. Tout le projet, dans la porosité faits-fiction qu'il met en place, dans son aspect complotiste ainsi que dans son intrusivité à l'égard du spectateur, tend à interroger cette illusion du contrôle. Cette dernière est par ailleurs renforcée non seulement par la structure en silo propre au transmédia, mais aussi par sa nature de dispositif. netwars prouve au spectateur à quel point il est démuni de toute emprise à l'égard de ses données personnelles, comme sont désemparés les gouvernements face aux menaces des cybercriminels en tout genre.

41 netwars agit à la façon d'un hack du spectateur, à l'image de notre hyperconnectivité contemporaine. C'est la nature transmédia de netwars qui permet que ce processus de hacking ait lieu et qui révèle toute la richesse de son univers et la complexité du projet. C'est elle qui construit les logiques de silo et de dispositif faisant vivre au spectateur une expérience unique. En naviguant au travers du transmédia, le spectateur se fait paranoïaque et comme le note très justement Nancy Huston dans L'Espèce fabulatrice, «La paranoïa, maladie de la surinterprétation, est la maladie congénitale de notre espèce » (2008: 83). Nous serions tous, nous, amateurs de fictions, des paranoïaques et netwars nous permet seulement d'assumer, de manière un peu plus explicite que d'autres projets artistiques, les plaisirs de la folie. 


\section{BIBLIOGRAPHIE}

Archibald, Samuel, 2011, « Vraie fiction et faux documents : notes éparses sur l'assassinat de Bill Gates ", Protée, vol. 39, n 1, p. 77-88, [En ligne], http://id.erudit.org/iderudit/1006729ar.

DOI : 10.7202/1006729ar

Barthes, Roland, 1970, S/Z, Paris, Seuil.

Borges, Jorge Luis, 1974, Fictions, nouv. éd. augm., Paris, Gallimard.

Brown, Gregory et Chiek, Yual, 2016, Leibniz on Compossibility and Possible Worlds, Cham, Springer.

Cohn, Dorrit, 2001, Le Propre de la fiction, trad. C. Hary-Schaeffer, Paris, Seuil.

Deleuze, Gilles, [1986] 2004, Foucault, Paris, Minuit.

Eco, Umberto, 1979, Lector in fabula. Le rôle du lecteur ou la coopération interprétative dans les textes narratifs, trad. M. Bouzaher, Paris, Grasset.

Ertzscheid, Olivier, 2012, « Des NTIC aux NTAD (nouvelles technologies de l'attention et de la distraction) $", 9^{e}$ congrès des enseignants documentalistes de l'Éducation nationale (Paris, 22-24 mars 2012), [En ligne], https://www.canal-u.tv/video/ fadben_federation_des_enseignants_documentalistes_de_l_education_nationale/ 1_organisation_des_connaissances_dans_le_contexte_du_web.9836, consulté le 12 juillet 2016. Foucault, Michel, 1975, Surveiller et punir. Naissance de la prison, Paris, Gallimard.

Foucault, Michel, 1976, Histoire de la sexualité 1 : la volonté de savoir, Paris, Gallimard.

Foucault, Michel, 1994a, Dits et écrits, 1954-1988. III, 1976-1979, éd. D. Defert et F. Ewald, Paris, Gallimard.

Foucault, Michel et Lagrange, Jacques, 1994b, « Le jeu de Michel Foucault : entretien avec des membres de la revue Ornicar (1977) », Dits et écrits, 1954-1988. 2, 1970-1975, éd. D. Defert et F. Ewald, Paris, Gallimard.

Genette, Gérard, 1991, Fiction et diction, Paris, Seuil.

Guelton, Bernard, 2007, Archifiction : quelques rapports entre les arts visuels et la fiction, Paris, Publications de la Sorbonne, coll. « Arts et monde contemporain ».

Groupierre, Karleen, 2012, Enjeux des transmédias de fiction en termes de création et de réception, Thèse de doctorat, Université Paris 8, [En ligne], karleen.fr/these.

Hamburger, Kate, 1973, Logic of Literature, trad. M. J. Rose, Bloomington, Indiana University Press. Huston, Nancy, 2008, L'Espèce fabulatrice, Paris, Actes Sud.

Iser, Wolfgang, 1985, L’Acte de lecture, théorie de l'effet esthétique, Bruxelles, Mardaga.

Jenkins, Henry, 2006, Convergence Culture: Where Old and New Media Collide, New York, New York University Press.

Kendall, Lewis Walton, 1990, Mimesis as Make-Believe: On the Foundations of the Representational Arts, Cambridge, MA, Harvard University Press.

Kristeva, Julia, 1969, Sèméiotikè. Recherches pour une sémanalyse, Paris, Seuil.

Lacan, Jacques, 1932, De la psychose paranoïaque dans ses rapports avec la personnalité, Paris, Seuil, coll. « Points. Essai ». 
Langner, Ralph, 2011, « Cracking Stuxnet, a 21st-century cyber weapon », Ted2011, [En ligne], https://www.ted.com/talks/ralph_langner_cracking_stuxnet_a_21st_century_cyberweapon, consulté le 2 mai 2017.

Lavocat, Françoise, 2016, Fait et fiction, Paris, Seuil.

Leibniz, Gottfried Wilhelm, [1765] 1993, Leibniz: New Essays on Human Understanding, éd.

P. Remnant et J. Bennett Cambridge, New York, Cambridge University Press.

Lewis, David, 1978, « Truth in Fiction », American Philosophical Quarterly, vol. 15, nº 1, p. 37-46.

Merzeau, Louise, 2014, « Le Flâneur impatient », Médium : Transmettre pour Innover, vol. 4, $\mathrm{n}^{\circ}$ 41, p. 20-29, [En ligne], https://halshs.archives-ouvertes.fr/halshs-01077637.

Merzeau, Louise, 2016, «Présence numérique : du mode d'existence transmédiatique », Colloque IMPEC (Lyon, 7 juillet 2016), [Support en ligne], http://merzeau.net/presence-numerique-dumode-dexistence-transmediatique/.

Pariser, Eli, 2011, The Filter Bubble: What the Internet Is Hiding from You, New York, Penguin Press.

Pavel, Thomas, 1988, Univers de la fiction, Paris, Seuil.

Rialland, Ivanne, 2008, «Le dispositif à l'œuvre », Compte rendu de P. Ortel (dir.), Discours, image, dispositif. Penser la représentation II, Paris, L’Harmattan, coll. « Champs visuels », [En ligne], consulté le 2 juin 2012.

Rech, Yannick et Triquet, Éric, 2012, « Le docufiction L'Odyssée de l'espèce. Analyse didactique et pistes d'exploitation en classe de terminale $\mathrm{S}$ », Revue de primatologie, $\mathrm{n}^{\circ} 4$, [En ligne], http:// primatologie.revues.org/1188.

DOI : $10.4000 /$ primatologie. 1188

Schaeffer, Jean-Marie, 1999, Pourquoi la fiction ?, Paris, Seuil.

Searle, John, [1972] 2009, Les Actes de langage, Paris, Hermann.

Searle, John, 1982, Sens et expression, Paris, Minuit.

Wachowski, Lilly et Lana, 1999, The Matrix, Warner Bros.

Weissberg, Jean-Louis, 1999, Présences à distance. Déplacement virtuel et réseaux numériques. Pourquoi nous ne croyons plus la télévision, Paris, L'Harmattan, coll. « Communication et civilisation ».

\section{Corpus}

Coleman, M. Sean, 2014, netwars - The Code 1: Crash (netwars 1 - A Cyber Crime Thriller), Bastei Entertainment, version Kindle.

Coleman, Sean, Mertikat, Felix, Klinke, Verena et al., 2015, The Butterfly Attack, filmtank GmbH, Application roman graphique.

Grotenhoff, Michael, 2015, netwars, filmtank GmbH. Documentaire TV.

Thiele, Lena, Miiqo Studios UG et al., 2015, netwars, filmtank GmbH. Web-documentaire.

\section{NOTES}

1. Nous utiliserons par commodité le terme de spectateur, mais il est important de noter que dans le cadre de la découverte d'un transmédia, ce dernier est tout à la fois lecteur et acteur. 
2. «Big brother is watching you and all that! " Toutes les traductions des extraits de netwars ont été effectuées par les autrices de l'article.

3. Nous utilisons le terme transmédia comme substantif ainsi que le font bon nombre de chercheurs et praticiens. Il s'agit de mettre en parallèle la pratique du transmédia, comme dispositif artistique et narratif, avec celle du film ou du livre, par exemple.

4. Stuxnet est un ver informatique découvert en 2010 qui aurait été conçu par la NSA et Israël afin d'attaquer les centrifugeuses d'enrichissement d'uranium iraniennes. Il s'agit du premier ver officiellement découvert qui ait espionné et soit intervenu au sein d'un système industriel à haut risque. Stuxnet fait partie de l'opération Olympic Games («Jeux olympiques»), une série de cyberattaques secrètes américaine contre le programme nucléaire iranien. Voir Langner (2011).

5. « He had been introduced to the Code, in its very early form, by a contact he met on one of the bulletin boards in the Deep Web. [...] He had been pulled up on it by a guy he assumed it was a guy who called himself the Salesman and the two had got chatting. [...] it was the Salesman who had taught him that a scattergun approach to leaking sensitive data did nothing to bring down governments, or make those responsible accountable for their actions and decisions. The Salesman dealt in a far more personal type of work on behalf of his clients, and slowly, over a matter of months he had recruited Strider to his way of thinking and turned an angry young cracker into a cold, calculating assassin. A professional. »

6. «Why I am doing this, I told you, money. [...] I'm just one guy, there is nothing I can do to change things. I'm just a salesman. What about you? Where are you in all of this my friend? I told you that we are in this together, right, that I won't let you down. Well, I lied, fuck, you are actually working for me. You are part of the botnet that I'm selling to the highest bidder. Establishing and exploit trust, remember. Take care now, thank you for your cooperation, and better luck next time.»

7. «Is it for real or some Hollywood prank?»

8. Traduit du web-documentaire, épisode 1 , «War: a human success story of evolution, it's what we do, it's in our nature like music or fucking », suivi de « Cyber war: the invisible threat ».

9. «You're already in deep, [...] you're an integral part of what happens next. »

10. «Let's see how vulnerable you really are?»

11. «Vous ne savez jamais qui a le contrôle». Notons que ces fichiers sont vides et n'engagent, en réalité, aucun risque pour l'ordinateur de l'utilisateur.

12. «+++ You just risked to give us access to all your data via drive-by download. +++ Not so cybersavvy after all. +++ And by the way, thanks for revealing all your strengths and weaknesses + ++ Don't call us, we'll call you. +++ »

13. « Friend ", « My friend ", « since we're friends ».

14. « Experts, who made them experts? [...] It is also advisable to make your own mind?»

15. "If you don't pay for the product, you are the product, everyone know that. Of course with all these informations they have on you, you do run the risk to being shut out of this beautiful and brand-new world, prevented from buying a new car, for example, because you broke the speed limit one too many times, or drop off from house care because your mother got sick one too many times, or thrown in jail because the taxman knows. "

16. Dans cette scène, Morpheus dialogue avec Néo : Morpheus : «Je suppose que pour l'instant tu te sens un peu comme Alice, tombée dans le terrier du lapin blanc. [...] Je le lis dans ton regard. Tu as le regard d'un homme prêt à croire tout ce qu'il voit, parce qu'il s'attend à s'éveiller à tout moment... Et paradoxalement ce n'est pas tout à fait faux. Crois-tu en la destinée Néo ? / Néo: Non. / Morpheus : Et pourquoi? / Néo : Parce que je n'aime pas l'idée de ne pas être aux commandes de ma vie. / [...] / Morpheus : Choisis la pilule bleue et tout s'arrête, après tu pourras faire de beaux rêves et penser ce que tu veux, choisis la pilule rouge tu restes au pays des merveilles et on descend avec le lapin blanc au fond du gouffre. N'oublie pas, je ne t'offre que la vérité, rien de plus. » (Wachowski 1999) 
17. Nous pensons ici par exemple à la pratique du webcam hacking. Profitant de failles notamment du plugin Adobe Flash dans Internet Explorer et Google Chrome, des pirates parviennent à mettre en marche à distance les micro et webcam des internautes à leur insu. Ces accès peuvent ensuite être monétisés, où dans le cas de personnes connues, les images et vidéo extraites peuvent être vendues.

18. Gabriel «Gabi » Siboni est un colonel des forces de défense de l'armée israélienne et directeur du programme militaire et stratégique, ainsi que de la cyber-guerre pour l'INSS (Institute for National Security Studies), centre de recherche israélien indépendant dédié à la sécurité nationale.

19. "You will never know me, but I will be the death of you. I saw what you did I see everything you do. I heard what you said I know your secrets. I know where you went I follow you everywhere. You are not above scrutiny I scrutinise everything. "

20. "We are all cut in the web of this wondrously seductive and evolving thing called interconnectedness. The Internet has finally succeeded in enlightening us with the knowledge which Buddhism has been trying to convey to us for over 2000 years: We are one, we are all connected. $»$

21. "A transmedia story unfolds across multiple media platforms with each new text making a distinctive and valuable contribution to the whole. In the ideal form of transmedia storytelling, each medium does what it does best-so that a story might be introduced in a film, expanded through television, novels, and comics; its world might be explored through game play or experienced as an amusement park attraction. »

\section{RÉSUMÉS}

Le projet netwars, consacré à l'univers de la cybercriminalité, est composé de cinq éléments porteurs : un documentaire TV, un web-documentaire, une application de récit interactif (roman graphique), une série de romans (audio, papier et e-book). netwars est un univers transmédia tel que défini par Henry Jenkins (2006), c'est-à-dire qu'il se déploie sur de multiples médias et chacun apporte une contribution significative à l'ensemble. netwars forme un univers trouble entre réalité et fiction dans lequel le spectateur se construit son propre récit et sa propre expérience en naviguant à son gré d'un média à un autre. Cette ambiguïté qui, comme nous le verrons, est génératrice de paranoïa, se voit thématisée, esthétisée et structurellement créée par le dispositif transmédia propre à netwars. Dans ce projet, la paranoïa ne provient pas tant du sujet en lui-même que du dispositif fictionnel qui mêle faits et éléments non référentiels ainsi que de la structure transmédia, dans sa dimension immersive et totalisante. Avec netwars tout est processus de hacking: le documentaire est hacké par la fiction, comme le spectateur par le dispositif transmédia.

The netwars project, dedicated to the world of cyber crime, is composed of five medias: a TV documentary, a web documentary, an interactive story app (Graphic Novel) and a series of novels (audio, paper and ebook). The netwars project is a transmedia universe as defined by Henry Jenkins (2006): it is spread over multiple media and each makes a significant contribution to the whole. netwars forms a blurred universe between reality and fiction in which the viewer builds his own story and experience navigating from one medium to another. This ambiguity, which, as we shall see, is generating paranoia, is aestheticized and structurally created by the transmedia 
quality of netwars. In this project, paranoia stands not so much in the subject itself than in the fictional device that mixes facts and non-referential elements, as well as in the transmedia structure in its immersive and totalizing dimension. With netwars everything is about hacking: fiction hacks the documentary just like the transmedia hacks the viewer.

INDEX

Mots-clés : transmédia, dispositif, silo, paranoïa, hack

Keywords : transmedia, dispositif, silo, paranoia, hack

\section{AUTEURS}

KARLEEN GROUPIERRE

Université Savoie Mont Blanc, LLSETI (EA 3706)

\section{ANAÏS GUILET}

Université Savoie Mont Blanc, LLSETI (EA 3706) 